\title{
Renormalized and renormalization-group invariant Hartree-Fock approximation
}

\author{
Claudio Destri* and Andrea Sartirana ${ }^{\dagger}$ \\ Dipartimento di Fisica dell'Università degli studi di Milano-Bicocca, \\ and INFN, Sezione di Milano, piazza della Scienza 3, I-20126 Milano, Italy
}

(Received 7 April 2005; published 6 September 2005)

\begin{abstract}
We study the renormalization problem for the Hartree-Fock approximation of the $O(N)$ invariant $\phi^{4}$ model in the symmetric phase and show how to systematically improve the corresponding diagrammatic resummation to achieve the correct renormalization properties of the effective field equations, including renormalization-group invariance with the one-loop beta function. These new Hartree-Fock dynamics is still of a mean-field type but includes memory effects which are generically nonlocal also in space.
\end{abstract}

DOI: 10.1103/PhysRevD.72.065003

PACS numbers: 11.10.Ef, 11.10.Gh, 11.15.Pg

\section{INTRODUCTION}

Cosmology, particle, and condensed matter physics have given, in recent years, a great impulse to the search of a deeper qualitative and quantitative understanding of outof-equilibrium dynamics of quantum fields. In fact, a treatment based on the first principle of the late time and strongly coupled evolution of quantum systems would provide a better insight in an important class of phenomena such as the reheating of the universe after inflation or thermalization of the quark gluon plasma in the ultrarelativistic heavy-ion and hadron colliders (RHIC, LHC).

The challenge of treating quantum field theories (QFT) in nonequilibrium conditions is that, except for a very short time, standard perturbation theory does not provide satisfactory results (this is true also at equilibrium with nonzero temperature). Therefore one has to look for nonperturbative approaches providing infinite partial resummations of Feynman diagrams [1,2]. The simplest of such schemes are the mean-field approximations such as the leading-order large- $N$ expansion [3-6] and the Hartree or Hartree-Fock (HF) variational method [7-10].

These approximations have been extensively studied and their features are well known [11-16]: they do provide a backreaction term on the evolution of quantum fluctuations that stabilize dynamics at late time but, on the other hand, they fail to properly describe an important aspect of late-time dynamics such as thermalization. More elaborate approaches going beyond mean field have been put forward by considering the 2-particle-irreducible (2PI) or the two-point-particle-irreducible effective action $[17,18]$ at two (or more) loops or at next-to-leading order in $1 / N$ expansion [19-22], yielding indeed approximate numerical thermalization at strong coupling.

Apart from the search for a better description of the latetime dynamics, there has recently been much progress in understanding more formal aspects of resummed approximations such as their renormalization properties. This is a long-standing problem $[23,24]$ and recently a systematic

\footnotetext{
*Email address: claudio.destri@mib.infn.it

${ }^{\dagger}$ Email address: andrea.sartirana@mib.infn.it
}

method has been developed [25-29] to remove divergences in the $\Phi$-derivable approximations based on applying a Bogoliubov-Parasiuk-Hepp-Zimmerman subtraction procedure to diagrams with resummed propagators.

In this article we will consider the simple HF approximation of the $O(N) \phi^{4}$ model as defined by variational principles, or equivalently by resummation of daisy (or bubble) diagrams. It is known $[8,23,24]$ that the usual renormalization of bare coupling and mass is not consistent, so that the simple Hartree approximation is not really renormalizable. We will show that this nonrenormalizability is due to the absence of leading logarithmically divergent contributions coming from diagrams which are not present in the standard HF resummation. We shall show that including also these contributions, plus suitably chosen finite parts, yields indeed a renormalized and renormalization-group (RG) invariant version of HF equations. We point out that these improved equations, although still of a mean-field type, are nonlocal in time (that is, there is memory) and generically nonlocal also in space, unlike the original ones. We also verify that all these nonlocalities disappear in the $N \rightarrow \infty$ limit, which is well known to provide a renormalizable, RG-invariant and local approximation to the out-of-equilibrium dynamics of the model.

Section II is dedicated to generalities. In Sec. II A the HF approach is introduced as a variational approximation with a Gaussian state functional in the Schrödinger picture. The corresponding well-known equations are rederived [see Eqs. (2.5) and (2.7)]. In Sec, II B we review the closed time path (CTP) formulation of out-of-equilibrium problems and the equivalent definition of the HF approximation as a resummation of bubble diagrams. In Sec. IIC we establish some relations between the two approaches and define a generating functional $\mathcal{F}^{\prime}$ [see Eqs. (2.20) and (2.21)] whose proper renormalization would render finite the dynamical problem.

In Sec. III we tackle the central problem of constructing renormalizable and RG-invariant HF-like equations. In Sec. III A we begin by studying, as a leading example, the static and homogeneous problem (i.e. the calculation of the effective potential) for the $N=1$ case of a single scalar field. As already stated above, our analysis leads naturally 
to add contributions from diagrams absent in the HF approximation, in such a way to include all leading logarithmic divergences and construct a modified effective potential [see Eqs. (3.22)] with the correct renormalization properties. In Sec. III B the analysis of the HF approximation and its renormalizable improvement are generalized to the fully dynamical and inhomogeneous $N>1$ case [see Eqs. (3.48)]. Further remarks on the freedom of choosing various initial conditions without spoiling renormalization and RG invariance are made in Sec. III C.

There are several possible developments along the lines of this work. First of all it would be interesting to study numerically our modified HF field equations, to investigate how the space-time nonlocalities affect the time evolution as compared to the standard HF approximation, which is known to fail even qualitatively at late times. Secondly, with the proper changes in the renormalization scheme, the derivation could be extended to the case of broken $O(N)$ symmetry, where the standard HF approximation faces other infrared difficulties connected with the Goldstone theorem [30]. Since our improved HF resummation adds also cutoff-independent contributions to the conventional one, it is conceivable that infrared properties of the model are affected too. Another challenging task is the extension of our approach to the full two-loop 2PI effective action, through the inclusion of the nonlocal sunset diagram which is absent by definition in any mean-field approximation. In fact one should expect that, even if such an inclusion allows one to recover renormalizability as compared to the conventional HF approximation, the two-loop 2PI self-consistent equations still lack RG invariance with the two-loop beta function, since the 2PI effective action does not contain all diagrams which contribute to the next-toleading ultraviolet divergences.

\section{GENERALITIES}

A nonequilibrium approach to quantum field theory is needed every time we have to deal with an initial value problem. In fact, the usual in-out formalism of quantum field theory (QFT) provides the means to calculate scattering expectation values

$$
\left\langle\text { out }\left|\mathcal{O}\left(t_{1}, t_{2}, \ldots\right)\right| \text { in }\right\rangle,
$$

where $\mid$ in $\rangle$ and $\mid$ out $\rangle$ represent particle states prepared at a distant past and future, respectively, while $\mathcal{O}$ is some observable depending on intermediate times $t_{1}, t_{2}, \ldots$ On the other hand, in order to study the real time dynamics from a given initial condition we need to know amplitudes like

$$
\left\langle\Psi\left(t_{0}\right)\left|\mathcal{O}\left(t_{1}, t_{2}, \ldots\right)\right| \Psi\left(t_{0}\right)\right\rangle,
$$

where $|\Psi\rangle$ is a generic state prepared at a initial time $t_{0}<$ $t_{1}, t_{2}, \ldots$ Out-of-equilibrium QFT provides the general setup for the calculation of such matrix elements, as well as more general expectation values in statistical mixtures of pure states like $|\Psi\rangle$.

In this section we briefly review some generalities about nonequilibrium QFT and point out some properties that will be useful to derive the results of the next section.

\section{A. Variational approach and HF approximation}

A simple and intuitive approach to the initial value problem is to treat QFT as ordinary quantum mechanics of extended systems. We restrict here to the case of interest for this paper: scalar field theory in $3+1$ dimensions with quartic interaction and unbroken $O(N)$ symmetry. The field variables are $\varphi_{i}(\boldsymbol{x})$ where $\boldsymbol{x}=\left(x_{1}, x_{2}, x_{3}\right)$ are space coordinates and $i=1, \ldots, N$ is the $O(N)$ index. Classical dynamics is defined by an action, functional of trajectories $\varphi_{i}(x)$, with $x=(x, t)$, in the form

$$
\begin{aligned}
S[\varphi]= & \int d^{4} x\left\{\frac{1}{2} \partial_{\mu} \varphi_{i}(x) \partial^{\mu} \varphi_{i}(x)-\frac{m^{2}}{2} \varphi_{i}(x) \varphi_{i}(x)\right. \\
& \left.-\frac{\lambda}{4 !}\left[\varphi_{i}(x) \varphi_{i}(x)\right]^{2}\right\},
\end{aligned}
$$

where $m^{2}$ and $\lambda$ are the squared mass and the coupling constant, respectively. In terms of $\varphi_{i}(\boldsymbol{x})$ and its canonical conjugated momentum $\pi_{i}(\boldsymbol{x})=\dot{\varphi}_{i}(\boldsymbol{x})$ the Hamiltonian reads

$$
\begin{aligned}
H[\varphi, \pi]= & \int d^{3} x\left[\frac{1}{2} \pi_{i}(\boldsymbol{x}) \pi_{i}(\boldsymbol{x})+\frac{1}{2} \nabla \varphi_{i}(\boldsymbol{x}) \cdot \nabla \varphi_{i}(\boldsymbol{x})\right. \\
& \left.+V\left(\varphi_{i}(\boldsymbol{x})\right)\right]
\end{aligned}
$$

where

$$
V(\varphi)=\frac{m^{2}}{2} \varphi_{i} \varphi_{i}+\frac{\lambda}{4 !}\left(\varphi_{i} \varphi_{i}\right)^{2} .
$$

Canonical quantization proceeds by imposing the standard commutation rules

$$
\left[\varphi_{j}(\boldsymbol{x}), \pi_{k}(\boldsymbol{y})\right]=i \delta_{j k} \delta^{3}(\boldsymbol{x}-\boldsymbol{y}) .
$$

In the "position" representation states are functionals of $\varphi_{i}(\boldsymbol{x})$ and the momentum operator reads

$$
\pi_{i}(x)=-i \frac{\delta}{\delta \varphi_{i}(x)} .
$$

It is then straightforward to write down the Hamiltonian operator and the corresponding Schrödinger equation.

In this framework, the standard variational methods of quantum mechanics suggest an easy nonperturbative approximation to dynamics (see e.g. [10,31,32]). The variational method follows from the observation that the Schrödinger equation is obtained by minimizing the Dirac action

$$
S_{\mathrm{D}}[\Psi]=\int d t\left\langle\Psi(t)\left|\left(i \partial_{t}-H\right)\right| \Psi(t)\right\rangle
$$


with respect to trajectories of vector states $|\Psi(t)\rangle$ in the Hilbert space. Therefore minimizing $S_{\mathrm{D}}$ over trajectories in a chosen variational family of states $\left|\Psi_{\text {var }}\right\rangle$ gives an approximate solution of the Schrödinger equation. In QFT, where scalar products are written as functional integrals, calculability strongly reduces the allowed family of states. Choosing states represented by Gaussian wave functionals, for which calculability is manifest, yields the HF approximation. Let us in fact consider the wave functional

$$
\begin{aligned}
\Psi[\varphi](t)= & \mathcal{N} \exp \{i\langle p(t) \mid \varphi-\phi(t)\rangle-\langle\varphi-\phi(t) \\
& \left.\left.\times\left|\left[\frac{1}{4} \mathcal{G}^{-1}(t)+i S(t)\right]\right| \varphi-\phi(t)\right\rangle\right\},
\end{aligned}
$$

where the variational parameters are the background field $\phi(x)$, the background momentum $p(x)$, the real symmetric positive kernel $G_{i j}(\boldsymbol{x}, \boldsymbol{y} ; t)$, and the real symmetric kernel $S_{i j}(\boldsymbol{x}, \boldsymbol{y} ; t)$ with the shorthand notation

$$
\langle a(t)|M(t)| b(t)\rangle \equiv \int d^{3} x d^{3} y a_{j}(\boldsymbol{x}, t) M_{j k}(\boldsymbol{x}, \boldsymbol{y} ; t) b_{k}(\boldsymbol{y}, t) .
$$

By evaluating $S_{\mathrm{D}}$ we obtain the variational action for these parameters

$$
\begin{aligned}
\Gamma_{\mathrm{HF}}[\phi, p, \mathcal{G}, S]= & \int d t\left(\left\langle p(t) \mid \dot{\phi}(t)-\frac{1}{2} p(t)\right\rangle\right. \\
& -\mathcal{V}[\mathcal{G}(t), \phi(t)]+\operatorname{Tr}[\dot{\mathcal{G}}(t) S(t) \\
& \left.-2 S(t) \mathcal{G}(t) S(t)-\frac{1}{8} G(t)^{-1}\right],
\end{aligned}
$$

where traces are taken over all indices and space variables, and

$$
\begin{aligned}
\mathcal{V}[\mathcal{G}, \phi]= & \frac{1}{2}\left\langle\phi\left|-\Delta+m_{0}^{2}\right| \phi\right\rangle+\frac{1}{2} \operatorname{Tr}\left[\left(-\Delta+m_{0}^{2}\right) G\right] \\
& +\int d^{3} x\left(\frac{\lambda_{0}}{4 !}\left(\phi_{i}(\boldsymbol{x}) \phi_{i}(\boldsymbol{x})\right)^{2}\right. \\
& +\frac{\lambda_{0}}{12} \phi_{i}(\boldsymbol{x}) \phi_{i}(\boldsymbol{x}) \mathcal{G}_{j j}(\boldsymbol{x}, \boldsymbol{x}) \\
& +\frac{\lambda_{0}}{6} \phi_{i}(\boldsymbol{x}) \phi_{j}(\boldsymbol{x}) \mathcal{G}_{i j}(\boldsymbol{x}, \boldsymbol{x}) \\
& \left.+\frac{\lambda_{0}}{4 !} \mathcal{G}_{i i}(\boldsymbol{x}, \boldsymbol{x}) \mathcal{G}_{j j}(\boldsymbol{x}, \boldsymbol{x})+\frac{\lambda_{0}}{12} \mathcal{G}_{i j}(\boldsymbol{x}, \boldsymbol{x}) \mathcal{G}_{i j}(\boldsymbol{x}, \boldsymbol{x})\right)
\end{aligned}
$$

Notice that the HF Dirac action is a first order action that involves only the first time derivative of fields and treats coordinates and momenta as independent variables. The equations of motion are obtained by the variation of Eq. (2.4) as

$$
\begin{aligned}
\ddot{\boldsymbol{\phi}}_{i}= & -\left\{\left[-\Delta+m_{0}^{2}+\frac{1}{6} \lambda_{0} \phi_{k} \boldsymbol{\phi}_{k}\right] \delta_{i j}\right. \\
& \left.+\frac{1}{2} \lambda_{0} \tau_{i j k m} \mathcal{G}_{k m}(\boldsymbol{x}, \boldsymbol{x})\right\} \boldsymbol{\phi}_{j}, \\
\dot{\mathcal{G}}_{i j}= & 2[G S+S G]_{i j}, \\
\dot{S}_{i j}(\boldsymbol{x}, \boldsymbol{y})= & {\left[\frac{1}{8} \mathcal{G}^{-2}-2 S^{2}\right]_{i j}(\boldsymbol{x}, \boldsymbol{y})-\frac{1}{2}\left[\left(-\Delta+m_{0}^{2}\right) \delta_{i j}\right.} \\
& \left.+\frac{1}{2} \lambda_{0} \tau_{i j k m}\left(\phi_{k} \phi_{m}+G_{k m}(\boldsymbol{x}, \boldsymbol{x})\right)\right] \delta^{3}(\boldsymbol{x}-\boldsymbol{y}),
\end{aligned}
$$

where

$$
\tau_{i j k m}=\frac{1}{3}\left(\delta_{i j} \delta_{k m}+\delta_{i k} \delta_{j m}+\delta_{i m} \delta_{j k}\right)
$$

and matrix multiplication, over both discrete and continuous indices, is understood. We have tacitly replaced in the last equations, and in general in the quantum Hamiltonian, the constant parameters $\lambda, m^{2}$ with bare (cutoff dependent) parameters $\lambda_{0}, m_{0}^{2}$. In fact the theory should be thought as regularized with an UV cutoff $\Lambda$ and then renormalized to remove the divergent dependence on $\Lambda$. We have not included here any field renormalization because, as is well known, this is absent in any mean-field-type approach such as the HF approximation.

To conclude this section we introduce an equivalent formulation of the Eqs. (2.5) in terms of mode functions $u_{\boldsymbol{k} a}[\boldsymbol{k}$ is the wave vector and $a$ the $O(N)$ polarization] that will be useful later on. For simplicity let us suppose that the initial $(t=0)$ kernels are transitionally invariant (while the background field and momentum may be point dependent). We can then write

$$
\begin{aligned}
& \mathcal{G}_{i j}(\boldsymbol{x}, \boldsymbol{y} ; 0)=\int \frac{d^{3} k}{(2 \pi)^{3}} \tilde{G}_{i j}(\boldsymbol{k}) e^{i \boldsymbol{k} \cdot \boldsymbol{x}}, \\
& S_{i j}(\boldsymbol{x}, \boldsymbol{y} ; 0)=\int \frac{d^{3} k}{(2 \pi)^{3}} \tilde{S}_{i j}(\boldsymbol{k}) e^{i \boldsymbol{k} \cdot \boldsymbol{x}} .
\end{aligned}
$$

Next, we introduce the $t=0$ mode functions by

$$
\begin{aligned}
& u_{\boldsymbol{k} a, i}(\boldsymbol{x}, 0)=\left[\tilde{\boldsymbol{G}}(\boldsymbol{k})^{1 / 2}\right]_{a i} e^{i \boldsymbol{k} \cdot \boldsymbol{x}}, \\
& \dot{u}_{\boldsymbol{k} a, i}(\boldsymbol{x}, 0)=\left[-\frac{i}{2} \tilde{\mathcal{G}}(\boldsymbol{k})^{-1}+2 S(\boldsymbol{k})\right]_{i j} u_{\boldsymbol{k} a, j}(\boldsymbol{x}, 0),
\end{aligned}
$$

and let them evolve according to the equations of motion

$$
\begin{aligned}
& \left\{\left(\square+m_{0}^{2}\right) \delta_{i j}+\frac{1}{2} \lambda_{0} \tau_{i j m n}\left[\phi_{m}(x) \phi_{n}(x)\right.\right. \\
& \left.\left.+\int \frac{d^{3} p}{(2 \pi)^{3}} u_{p b, m}(x) \bar{u}_{p b, n}(x)\right]\right\} u_{\boldsymbol{k} a, j}(x)=0 .
\end{aligned}
$$

Then one can show that the last two equations in Eqs. (2.5) are equivalent to

$$
\begin{aligned}
\mathcal{G}_{i j}(\boldsymbol{x}, \boldsymbol{y} ; t) & =\int \frac{d^{3} k}{(2 \pi)^{3}} u_{\boldsymbol{k} a, i}(\boldsymbol{x}, t) \bar{u}_{\boldsymbol{k} a, j}(\boldsymbol{y}, t), \\
\dot{u}_{\boldsymbol{k} a, i}(\boldsymbol{x}, t)= & \int d^{3} y\left\{-\frac{i}{2}\left[\mathcal{G}^{-1}\right]_{i j}(\boldsymbol{x}, \boldsymbol{y} ; t)\right. \\
& \left.+2 S_{i j}(\boldsymbol{x}, \boldsymbol{y} ; t)\right\} u_{\boldsymbol{k} a, j}(\boldsymbol{y}, t) .
\end{aligned}
$$

Through the mode functions we can introduce the symmetric correlation

$$
G_{i j}(x, y)=G_{j i}(y, x)=\operatorname{Re} \int \frac{d^{3} k}{(2 \pi)^{3}} u_{\boldsymbol{k} a, i}(x) \bar{u}_{\boldsymbol{k} a, j}(y)
$$

whose equal time value reproduces the kernel $G$,

$$
\left.G_{i j}(x, y)\right|_{x_{0}=y_{0}=t}=G_{i j}(\boldsymbol{x}, \boldsymbol{y} ; t) .
$$

Then we can reformulate the dynamics in terms of $\phi$ and $G$ 
in a manifestly covariant way as

$$
\begin{gathered}
\left\{\left[\square+m_{0}^{2}+\frac{1}{6} \lambda_{0} \phi_{k}(x) \phi_{k}(x)\right] \delta_{i j}\right. \\
\left.+\frac{1}{2} \lambda_{0} \tau_{i j k m} G_{k m}(x, x)\right\} \phi_{j}(x)=0, \\
\left\{\left(\square+m_{0}^{2}\right) \delta_{i j}+\frac{1}{2} \lambda_{0} \tau_{i j k m}\left[\phi_{k}(x) \phi_{m}(x)\right.\right. \\
\left.\left.+G_{k m}(x, x)\right]\right\} G_{i j}(x, y)=0 .
\end{gathered}
$$

\section{B. CTP formalism and resummations}

The general approach to nonequilibrium problems in QFT was developed by Keldish and Schwinger. It is known as CTP formalism and allows one to use standard path integral functional methods (see [17,18,33,34]). Basically it is obtained by introducing path integrals on a time path going from $t=0$ to $t=+\infty$ and back. Field integration variables are then doubled and subdivided into $(+)-$ components, for the path integral forward in time, and $(-)-$ components for the backward piece. Given an initial state defined by the functional $\Psi[\varphi]$ (in our case it will be a Gaussian state) one writes down the functional integral

$$
\begin{aligned}
e^{i \mathcal{W}\left[j_{+}, j_{-}\right]}= & \int \mathcal{D} \varphi_{+} \mathcal{D} \varphi_{-} \Psi\left[\varphi_{+}\right] \\
& \times \bar{\Psi}\left[\varphi_{-}\right] e^{i S\left[\varphi_{+}\right]-i S\left[\varphi_{-}\right]+i\left\langle j_{+} \mid \varphi_{+}\right\rangle-i\left\langle j_{-} \mid \varphi_{-}\right\rangle},
\end{aligned}
$$

where $S$ is the classical action (2.1) and we have used the notation (2.3) for the current terms. Integration is on trajectories from $t=0$ to $t=+\infty$ (with the condition $\varphi_{+}=$ $\varphi_{-}$at $\left.t=+\infty\right)$ and $\varphi_{ \pm}$in the wave functional is the $t=0$ section of $\varphi_{ \pm}$. By construction $\mathcal{W}\left[j_{+}, j_{-}\right]$is the generating functional of connected Green functions

$$
\begin{aligned}
& G_{+} \ldots+-\cdots-\left(x_{1}, \ldots, x_{n}, y_{1}, \ldots, y_{n}\right) \\
& \left.\quad \equiv \frac{(-i)^{n+m} \delta^{n+m} \mathcal{W}}{\delta j_{+}\left(x_{1}\right) \cdots \delta j_{+}\left(x_{n}\right) \delta j_{-}\left(y_{1}\right) \cdots \delta j_{-}\left(y_{m}\right)}\right|_{j_{+}=0 j_{-}=0} \\
& \quad=-i\left\langle\Psi\left|\overline{\mathcal{T}}\left\{\varphi\left(y_{1}\right) \cdots \varphi\left(y_{m}\right)\right\} \mathcal{T}\left\{\varphi\left(x_{1}\right) \cdots \varphi\left(x_{n}\right)\right\}\right| \Psi\right\rangle_{\mathrm{conn}},
\end{aligned}
$$

where $\mathcal{T}$ and $\overline{\mathcal{T}}$ define time ordered and inverse ordered products, respectively, and internal indices have been omitted for ease of notation. The effective action $\Gamma_{1 \mathrm{PI}}\left[\phi_{+}, \phi_{-}\right]$, which is the generator of 1PI vertex functions, is the Legendre transform of $\mathcal{W}\left[j_{+}, j_{-}\right]$from the currents $j_{ \pm}$ to the fields $\phi_{ \pm}$. The equation of motion for the background field $\phi(x)=\langle\Psi|\varphi(x)| \Psi\rangle$ then reads

$$
\left.\frac{\delta \Gamma_{1 \mathrm{PI}}}{\delta \phi_{+}(x)}\right|_{\phi_{+}=\phi_{-}=\phi}=0 .
$$

Notice that, in the case of the Gaussian wave functional equation (2.2), $\Gamma_{1 \mathrm{PI}}$ depends parametrically only on the $t=$ 0 kernels $G(0)$ and $S(0)$, while the $t=0$ background field $\phi(0)$ and $p(0)$ enter, instead, as initial conditions for the equation (2.11).
The perturbative diagrammatic expansion in the CTP formalism proceeds as in vacuum QFT in terms of free propagators

$$
\begin{aligned}
& G_{++}^{0}(x, y) \equiv G_{F}^{0}(x, y)=-\left.i\langle\Psi|\mathcal{T} \varphi(x) \varphi(y)| \Psi\rangle_{\mathrm{conn}}\right|_{\lambda=0}, \\
& G_{--}^{0}(x, y) \equiv G_{\bar{F}}^{0}(x, y)=-\left.i\langle\Psi|\overline{\mathcal{T}} \varphi(x) \varphi(y)| \Psi\rangle_{\mathrm{conn}}\right|_{\lambda=0}, \\
& G_{+-}^{0}(x, y)=G_{-+}^{0}(y, x)=-\left.i\langle\Psi|\varphi(y) \varphi(x)| \Psi\rangle_{\mathrm{conn}}\right|_{\lambda=0}
\end{aligned}
$$

and vertices (for clarity, we write here explicitly the internal indices)

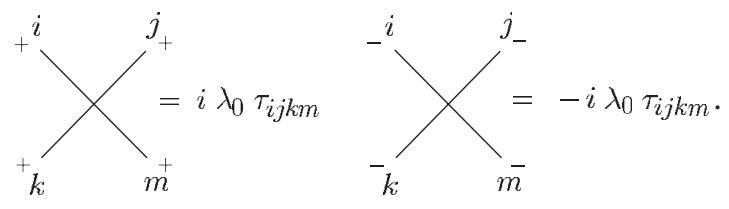

It can be shown that, in generic out-of-equilibrium contexts or even at equilibrium with nonzero temperature, plain perturbation theory is of very little help and many resummation methods have been developed to go beyond it. A very successful instrument in this sense is the $2 \mathrm{PI}$ effective action (see $[17,18])$. It is defined as the double Legendre transform of the $\mathcal{W}$ generating functional with respect to the usual current one-point $j_{ \pm}$and to the two-points current $K_{\alpha \beta}(x, y)$ coupled through the term

$$
\frac{1}{2} \int d^{4} x \int d^{4} y K_{\alpha \beta}(x, y) \varphi_{\alpha}(x) \varphi_{\beta}(x),
$$

where $\alpha, \beta= \pm . \Gamma_{2 \mathrm{PI}}$ is a functional of the classical fields $\phi_{\alpha}$ and of the propagators $G_{\alpha \beta}$. It yields two equations of motions

$$
\left.\frac{\delta \Gamma_{2 \mathrm{PI}}}{\delta \phi_{\alpha}(x)}\right|_{*}=0,\left.\quad \frac{\delta \Gamma_{2 \mathrm{PI}}}{\delta G_{\alpha \beta}(x, y)}\right|_{*}=0
$$

Here the notation $\left.\right|_{*}$ indicates that, by their physical meaning, the $( \pm)$-component fields and propagators have to satisfy, on the solutions of motion, the following relations:

$$
\begin{aligned}
& \phi_{-}(x)=\phi_{-}(x)=\phi(x), \\
& G_{F}(x, y)=G_{+-}(y, x) \theta\left(x_{0}-y_{0}\right)+G_{+-}(x, y) \theta\left(y_{0}-x_{0}\right), \\
& G_{\bar{F}}(x, y)=G_{+-}(x, y) \theta\left(x_{0}-y_{0}\right)+G_{+-}(y, x) \theta\left(y_{0}-x_{0}\right) .
\end{aligned}
$$

Hence the system (2.13) reduces to two coupled equations for $\phi$ and $G_{+-}$only. Moreover, any initial Gaussian state may be absorbed in the $t=0$ term for the $j_{\alpha}$ and $K_{\alpha \beta}$ currents, so that, by the double Legendre transform the initial Gaussian state disappears from the effective action, but fixes the initial conditions on $\phi$ and $G_{+-}$. The role of $\phi(0)$ and $p(0)=\dot{\phi}(0)$ is immediate, while for the kernels we have 


$$
\begin{aligned}
\left.G_{+-}(x, y)\right|_{x_{0}=y_{0}=0}= & -i\langle\Psi|\varphi(\boldsymbol{y}) \varphi(\boldsymbol{x})| \Psi\rangle \\
& -i \phi(\boldsymbol{y}, 0) \phi(\boldsymbol{x}, 0) \\
= & G(\boldsymbol{x}, \boldsymbol{y} ; 0), \\
\left.\frac{\partial}{\partial y_{0}} G_{+-}(x, y)\right|_{x_{0}=y_{0}=0}= & -i\langle\Psi|\pi(\boldsymbol{y}) \phi(\boldsymbol{x})| \Psi\rangle \\
& -i p(\boldsymbol{y}, 0) \phi(\boldsymbol{x}, 0) \\
= & 2 i[G S](\boldsymbol{x}, \boldsymbol{y} ; 0)+\frac{1}{2} \delta^{3}(\boldsymbol{x}-\boldsymbol{y}) .
\end{aligned}
$$

Given $\Gamma_{2 \mathrm{PI}}$ at a certain perturbative loop order, if we solve the second equation in (2.13) for a generic $\phi$ and substitute the result $G[\phi]$ into the first one we obtain the background equations of motion corresponding to a resummed diagrammatic approximation of the 1PI effective action $\Gamma_{1 \mathrm{PI}}$.

For a scalar theory, the $\Gamma_{2 \mathrm{PI}}$ has the general form

$$
\begin{aligned}
\Gamma_{2 \mathrm{PI}}[\phi, G]= & S[\phi]+\frac{i}{2} \operatorname{Tr}[\log G]+\frac{i}{2} \operatorname{Tr}\left[G_{0}^{-1} G\right] \\
& +\Gamma_{2}[\phi, G] .
\end{aligned}
$$

Here $S$ is the complete classical action of the double time path (i.e. $S=S_{+}-S_{-}$). Traces are taken over all indices $i, \alpha$ and $x . G_{0}^{-1}$ is the second derivative of the action in a $\phi$ background, $\Gamma_{2}$ is the sum of all vacuum 2PI diagrams with $G$ propagators and vertices defined by the classical action in a $\phi$ background. To two loops level the diagrams contributing to the $\Gamma_{2}$ are the " 8 " and "sunset" diagrams

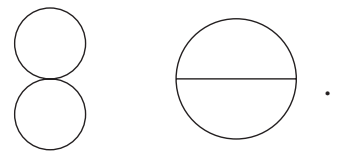

The HF approximation corresponds to consider only the first contribution to $\Gamma_{2}$.

$$
\Gamma_{2}=\frac{i}{8} \lambda_{0}\left[G_{F}^{2}(x, x)-G_{\bar{F}}^{2}(x, x)\right] .
$$

In fact using this form of $\Gamma$ in the field equations (2.13), setting $G(x, y)=\frac{i}{2}\left[G_{-+}(x, y)+G_{-+}(y, x)\right]$ and observing that the antisymmetric combination decouples, one obtains exactly the HF equations (2.9).

Notice that " 8 " is the only $2 \mathrm{PI}$ diagram made of a "product" of loops corresponding to a mean-field contribution to the mass. In the 1PI framework this corresponds to a resummation of all vacuum 1PI diagrams with daisy and superdaisy topologies of the form
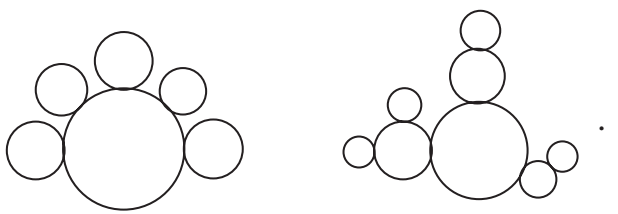

\section{Physical representation and reparametrization of HF effective action}

In this section we derive useful explicit relations connecting the diagrammatic definition of $\Gamma_{\mathrm{HF}}$ to the variational approach of Sec. II A. To this purpose it is convenient to introduce a different representation of the CTP formalism, known as the physical representation (see [34]). We introduce the field redefinitions (omitting again the internal indices to simplify notation)

$$
\phi_{\Delta}=\phi_{+}-\phi_{-}, \quad \phi_{c}=\frac{1}{2}\left(\phi_{+}+\phi_{-}\right)
$$

and write the 1PI effective action as a functional of these new fields, $\Gamma_{1 \mathrm{PI}}=\Gamma\left[\phi_{\Delta}, \phi_{c}\right]$. By calculating vertex functions, one then finds

$$
\left.\frac{\delta^{n} \Gamma}{\delta \phi_{c}\left(x_{1}\right) \cdots \delta \phi_{c}\left(x_{n}\right)}\right|_{\phi_{\Delta}=0}=0
$$

and

$$
\left.\frac{\delta^{n+m} \Gamma}{\delta \phi_{c}\left(x_{1}\right) \cdots \delta \phi_{c}\left(x_{n}\right) \delta \phi_{\Delta}\left(y_{1}\right) \cdots \delta \phi_{\Delta}\left(y_{m}\right)}\right|_{\phi_{\Delta}=0}=0
$$

if the time component of any one of the $x$ 's is larger than the time component of any one of the $y$ 's. Let us also remark that, by the definition of the CTP generating functional, all time coordinates in the vertex functions are supposed to be positive so we can set, as well, these functions to be zero for any negative time. Using Eq. (2.16) together with Eq. (2.11) one can write the equations of motions in the form

$$
\left.\frac{\delta \Gamma}{\delta \phi_{\Delta}}\right|_{\phi_{\Delta}=0, \phi_{c}=\phi}=0 .
$$

Notice that $2 n$-legs vertex functions with one $\phi_{\Delta}$ leg and $2 n-1 \phi_{c}$ legs are the only ones contributing to these equations of motion. Then Eq. (2.17) guarantees that all the terms nonlocal in time in Eq. (2.18) do satisfy causality.

Perturbative calculations by diagrammatic expansion in the physical representation are based on the free propagators

$$
\begin{aligned}
G_{c \Delta}^{0}(x, y) & \equiv G_{A}^{0}(x, y) \\
& =-\left.i \theta\left(y_{0}-x_{0}\right)\langle\Psi|[\phi(x), \phi(y)]| \Psi\rangle\right|_{\lambda=0}, \\
G_{\Delta c}^{0}(x, y) & \equiv G_{R}^{0}(x, y) \\
& =-\left.i \theta\left(x_{0}-y_{0}\right)\langle\Psi|[\phi(x), \phi(y)]| \Psi\rangle\right|_{\lambda=0}, \\
G_{\Delta \Delta}^{0}(x, y) & =-\left.2 i\langle\Psi|\{\phi(y), \phi(x)\}| \Psi\rangle_{\text {conn }}\right|_{\lambda=0}, \\
G_{c c}^{0}(x, y) & =0 .
\end{aligned}
$$

The free retarded and advanced Green functions $G_{A}^{0}$ and $G_{R}^{0}$ do not depend on the initial state and are translational invariant. The correlation function $G=\frac{i}{4} G_{\Delta \Delta}^{0}$, instead, 
does depend on $|\Psi\rangle$. The vertices are
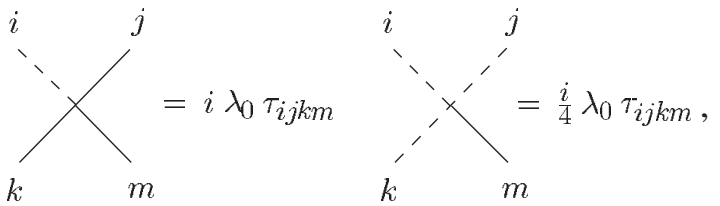

where solid lines represent $\phi_{c}$ legs while dotted lines represent $\phi_{\Delta}$ legs.

As stated above, the HF approximation consists in the resummation of diagrams with daisy and superdaisy topologies. Therefore the functional differentiations which produce the vertex functions act only on propagators; hence the diagrams contributing to a $2 n$-legs vertex function (both in \pm and physical representations) must have the form

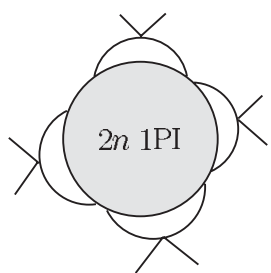

that is, the $2 n$ legs are pairwise connected to $n$ vertices. This means that the HF effective action can be written in the form

$$
\Gamma_{\mathrm{HF}}\left[\phi_{\Delta}, \phi_{c}\right]=-\left\langle\phi_{\Delta}|\square| \phi_{c}\right\rangle-\mathcal{F}[\xi, \chi, \eta],
$$

where $\mathcal{F}$ is a functional of the following composite matrix fields:

$$
\begin{gathered}
\xi_{i j}(x)=\phi_{c, i}(x) \phi_{c, j}(x), \quad \chi_{i j}(x)=\phi_{c, i}(x) \phi_{\Delta, j}(x), \\
\eta_{i j}(x)=\phi_{\Delta, i}(x) \phi_{\Delta, j}(x) .
\end{gathered}
$$

We now introduce, for ease of notation, the new object

$$
\mathcal{F}^{\prime}[\xi]_{i j}(x)=\left.\frac{1}{2} \frac{\delta \mathcal{F}}{\delta \chi_{i j}(x)}\right|_{\chi=\eta=0}
$$

which is a functional of $\xi_{i j}(x)=\phi_{c, i}(x) \phi_{c, j}(x)=$ $\phi_{i}(x) \phi_{j}(x)$ only. Then the equation of motion in the HF approximation takes the general form

$$
\left(\square \delta_{i j}+2 \mathcal{F}_{i j}^{\prime}\right) \phi_{j}=0 .
$$

By comparison with Eqs. (2.5) and (2.8) we obtain the following expression for $\mathcal{F}^{\prime}$ :

$$
\begin{aligned}
\mathcal{F}^{\prime}[\xi]_{i j}(x)= & \frac{m_{0}^{2}}{2} \delta_{i j}+\frac{\lambda_{0}}{12} \delta_{i j} \xi_{k k}(x) \\
& +\frac{\lambda_{0}}{4} \tau_{i j}^{k m} \int \frac{d^{3} p}{(2 \pi)^{3}} u_{p a, i}(x) \bar{u}_{p a, j}(x) .
\end{aligned}
$$

The dependence of the mode functions $u_{p a}$ on $\xi$ is fixed by solving Eq. (2.7) for a generic $\phi$ field. Notice that the identification of Eq. (2.21) is somewhat arbitrary since we can add to $\mathcal{F}^{\prime}$ a generic functional term $A[\xi]_{i j k m} \times$ $(x) \xi_{k m}(x)$ with $A_{i j k m}=-A_{j k i m}$ without changing the contribution of $\mathcal{F}^{\prime}$ to the effective action and to the equations of motion (2.20).

The role of the functional $\mathcal{F}^{\prime}$ in the generation of vertex functions can be easily established. Let us consider the $2 n$-points vertex function with one $\phi_{\Delta}$ field leg and $m(=$ $2 n-1) \phi_{c}$ field legs (as we said those we are interested in for they contribute to the equations of motion), a simple calculation leads to

$$
\begin{aligned}
& \Gamma_{i \mid j_{1} \cdots j_{m}}^{(2 n)}\left(x \mid y_{1}, \ldots, y_{m}\right) \\
& \equiv\left.\frac{\delta^{2 n} \Gamma}{\delta \phi_{\Delta i}(x) \delta \phi_{c j_{1}}\left(y_{1}\right) \cdots \delta \phi_{c j_{m}}\left(y_{m}\right)}\right|_{\phi_{\Delta}=0 \phi_{c}=0} \\
&= \square \delta^{4}\left(x-y_{j_{1}}\right) \delta_{1, n}+\frac{2}{(n-1) !} \sum_{p \in \Sigma_{m}} \delta^{4}\left(x-p y_{1}\right) \\
& \times\left[\prod_{k=1}^{n-1} \delta^{4}\left(p y_{2 k}-p y_{2 k+1}\right)\right] \\
& \times\left.\frac{\delta^{n-1} \mathcal{F}^{\prime}[\xi]_{i p j_{1}}(x)}{\delta p \xi_{j_{2} j_{3}}\left(y_{2}\right) \cdots \delta p \xi_{j_{m-1} j_{m}}\left(y_{m}\right)}\right|_{\xi=0}, \quad(2.22)
\end{aligned}
$$

where $\Sigma_{m}$ is the set of all permutations on $\{1 \cdots m\}$ and we used the notation $p y_{i} \equiv y_{p(i)}, p j_{i} \equiv j_{p(i)}$, and $p \xi_{j_{i} j_{k}}\left(y_{s}\right) \equiv$ $\xi_{p j_{i} p j_{k}}\left(p y_{s}\right)$.

Let us observe that the general structure of the $2 n$-points vertex functions in Eq. (2.22) is a direct consequence of the mean-field form (2.20) of the equations of motion, regardless of the specific form of the functional $\mathcal{F}^{\prime}[\xi]$. In this sense it is more general than the HF approximation, holding true for any mean-field-type dynamics.

In what follows we are going to study the problem of renormalizability (and RG invariance) of the effective field equations in the HF approximation. By the results of this section we need to consider only the vertex functions contributing to the equations of motion and hence only the functional $\mathcal{F}^{\prime}$.

\section{RENORMALIZABILITY AND RG INVARIANCE}

We are concerned here about the possibility of constructing a set of HF equations in which all the divergent dependence from the cutoff has been removed by a suitable renormalization procedure and, at the same time, no dependence on the renormalization scale has been introduced. In brief we are going to study the problem of renormalizability and $\mathrm{RG}$ invariance of $\mathrm{HF}$ equations. This will lead us to some results that go beyond the strict mean-field approximation and include resummations coming from 2PI effective action loop expansion.

In this section we will deal with the general case of an inhomogeneous (in space) dynamical problem within the $O(N)$ symmetric scalar field theory with unbroken symmetry. However the main aspects of the problem can be 
more easily pointed out by considering a simpler case. In the first subsection, therefore, we restrict to the static problem, that is the calculation of the effective potential, for the $N=1$ theory.

\section{A. A simple instructive example: effective potential for $N=1$}

We want to study the renormalization problem of the static and translationally invariant version of Eqs. (2.5) when $N=1$. This situation follows, in the variational approach of Sec. II A, by considering a field $\phi$ constant throughout space-time, with vanishing momentum $p$, and a constant translationally invariant $G$ kernel

$$
\boldsymbol{G}(\boldsymbol{x}-\boldsymbol{y})=\int \frac{d^{3} k}{(2 \pi)^{3}} \tilde{\mathcal{G}}(\boldsymbol{k}) e^{-i \boldsymbol{k}(\boldsymbol{x}-\boldsymbol{y})}
$$

with $S=0$. By a simple substitution in Eq. (2.5) we obtain

$$
\begin{aligned}
\frac{1}{4 \tilde{G}^{2}(\boldsymbol{k})} & =\boldsymbol{k}^{2}+m_{0}^{2}+\frac{\lambda_{0}}{2} \boldsymbol{\phi}^{2}+\frac{\lambda_{0}}{2} \int_{|\boldsymbol{p}|<\Lambda} \frac{d^{3} p}{(2 \pi)^{3}} \tilde{G}(\boldsymbol{p}), \\
0 & =\left[m_{0}^{2}+\frac{\lambda_{0}}{6} \boldsymbol{\phi}^{2}+\frac{\lambda_{0}}{2} \int_{|\boldsymbol{p}|<\Lambda} \frac{d^{3} p}{(2 \pi)^{3}} \tilde{\mathcal{G}}(\boldsymbol{p})\right] \boldsymbol{\phi},
\end{aligned}
$$

where a sharp cutoff $\Lambda$ has been introduced as regularization.

Equivalently we can consider the equations of motion as defined by the 1PI effective action and reduce to the static case by evaluating $\Gamma_{1 \text { PI }}$ on a space-time constant $\phi$. So letting

$$
\hat{V}^{\prime}(\xi)=\left.\mathcal{F}^{\prime}[\xi]\right|_{\xi=\text { const }},
$$

we can define the effective potential by $V_{\text {eff }}(\phi)=\hat{V}\left(\phi^{2}\right)$, with $\hat{V}(\xi)$ the primitive of $\hat{V}^{\prime}(\xi)$ vanishing at $\xi=0$. From Eq. (2.20) the equation for the background field $\phi$ is

$$
V_{\text {eff }}^{\prime}(\phi)=2 \hat{V}^{\prime}\left(\phi^{2}\right) \phi=0 .
$$

Comparison with the second equation in (3.1) leads immediately to

$$
\hat{V}^{\prime}(\xi)=\frac{m_{0}^{2}}{2}+\frac{\lambda_{0}}{12} \xi+\frac{\lambda_{0}}{4} \int_{|\boldsymbol{p}|<\Lambda} \frac{d^{3} p}{(2 \pi)^{3}} \tilde{G}(\boldsymbol{p}),
$$

where the implicit dependence of $\tilde{\mathcal{G}}$ on $\xi=\phi^{2}$ is obtained by solving the first Eq. (3.1) with a generic $\phi$. We recall also that $V_{\text {eff }}(\phi)$ is the generator of vertex functions with all incoming momenta set to zero (for constant homogeneous fields the distinction between CTP and vacuum standard formalism is immaterial).

As usual, one introduces the ansatz

$$
\tilde{G}(k)=\frac{1}{2 \sqrt{k^{2}+M^{2}}}
$$

that allows one to cast the first of Eqs. (3.1) in the form of a mass-gap equation

$$
M^{2}(\xi)=m_{0}^{2}+\frac{\lambda_{0}}{2} \xi+\frac{\lambda_{0}}{2} \int_{p^{2}<\Lambda} \frac{d^{4} p}{(2 \pi)^{4}} \frac{1}{p^{2}+M^{2}(\xi)},
$$

where the integration has been written in four Euclidean dimensions $\left(p^{2}=p_{0}^{2}+p^{2}\right)$. By comparing Eq. (3.3) with Eq. (3.2) we can rewrite the latter as

$$
\hat{V}^{\prime}(\xi)=\frac{M^{2}(\xi)}{2}-\frac{\lambda_{0}}{6} \xi
$$

so that, by combining Eqs. (3.3) and (3.4), we have

$$
\begin{aligned}
\hat{V}^{\prime}(\xi)= & \frac{m_{0}^{2}}{2}+\frac{\lambda_{0}}{12} \xi+\frac{\lambda_{0}}{4} \int_{p^{2}<\Lambda^{2}} \frac{d^{4} p}{(2 \pi)^{4}} \\
& \times \frac{1}{p^{2}+2 \hat{V}^{\prime}(\xi)+\frac{1}{3} \lambda_{0} \xi} .
\end{aligned}
$$

This is a self-consistent definition of the effective potential and is the central equation that we are going to use in this subsection.

Let us apply the standard renormalization procedure. We introduce two physical parameters, the renormalized squared mass $m^{2}$ and the coupling constant $\lambda$, which are identified, respectively, as the second and fourth derivatives of the effective potential at zero field, so that

$$
\begin{gathered}
\left.\frac{d^{2} V_{\text {eff }}}{d \phi^{2}}\right|_{\phi=0}=2 \hat{V}^{\prime}(0)=m^{2}, \\
\left.\frac{d^{4} V_{\text {eff }}}{d \phi^{4}}\right|_{\phi=0}=12 \hat{V}^{\prime \prime}(0)=\lambda .
\end{gathered}
$$

These are the two renormalization conditions that, once inverted, determine the dependence of the bare parameters $\lambda_{0}(\lambda, m, \Lambda)$ and $m_{0}^{2}(\lambda, m, \Lambda)$ on the physical ones and the cutoff. The assumed existence and positivity of $2 \hat{V}^{\prime}(0)$ provides the definition of unbroken symmetry, which is our choice here.

Using Eq. (3.5), we can calculate the explicit HF form of Eq. (3.6)

$$
\begin{aligned}
& m^{2}=m_{0}^{2}+\frac{\lambda_{0}}{2} I_{E}^{(1)}\left(m^{2}, \Lambda\right), \\
& \lambda=\frac{3 \lambda_{0}}{1+\frac{1}{2} \lambda_{0} I_{E}^{(2)}\left(m^{2}, \Lambda\right)}-2 \lambda_{0},
\end{aligned}
$$

where, to shorten notation, we have introduced

$$
I_{E}^{(n)}\left(m^{2}, \Lambda\right)=\int_{p^{2}<\Lambda^{2}} \frac{d^{4} p}{(2 \pi)^{4}} \frac{1}{\left(p^{2}+m^{2}\right)^{n}}
$$

and the suffix $E$ refers to the Euclidean form of the integral. As $\Lambda \rightarrow \infty$ one sees that $I_{E}^{(1)}$ is quadratically divergent in $\Lambda$, while $I_{E}^{(2}$ is logarithmically divergent and all other $I_{E}^{(n)}$ are finite.

We now point out the problems that occur with this standard procedure. 
First of all we can see that there is a pathological behavior of the effective quartic coupling $\lambda$ as a function of the bare parameter $\lambda_{0}$ at fixed cutoff $\Lambda$. In fact $\lambda$ has the correct 1-loop $\lambda_{0}^{2}$ term dictated by perturbation theory, but certainly fails at higher orders, since it exhibits an unphysical behavior, growing to a maximum value at $\lambda_{0}=\lambda_{0}^{\max }$, then decreasing to zero and to even more unphysical negative values. This implies the breakdown of the HF approximation for values of $\lambda_{0}$ greater than $\lambda_{0}^{\max }$ in a theory at fixed cutoff. Another way of looking at this problem is to invert Eqs. (3.7) to obtain $m_{0}^{2}$ and $\lambda_{0}$ as functions of $\Lambda$ parametrized by $m^{2}$ and $\lambda$. A full trajectory in $\Lambda$ of $m_{0}^{2}$ and $\lambda_{0}$ corresponds to a single renormalized theory describing the dynamics at momentum scales much smaller than $\Lambda$. Clearly we must restrict our attention to the monotonically increasing branch through the origin at $\lambda_{0}=\lambda=0$. But these trajectories, for any positive value of $\lambda$, exhibit a "Landau obstruction" at the value of $\Lambda$ which corresponds to $\lambda_{0}^{\max }$. This obstruction is even more troublesome than the Landau pole present in the standard 1-loop-renormalization-group (1LRG) improved relation, which by a suitable choice of finite parts can be written as

$$
\left.\lambda_{0}(\lambda, \Lambda / m)\right|_{1 \mathrm{LRG}}=\frac{\lambda}{1-\frac{3}{2} \lambda I_{E}^{(2)}\left(m^{2}, \Lambda\right)}
$$

in terms of the whole $I_{E}^{(2)}\left(m^{2}, \Lambda\right)$ rather than just its leading divergence $\frac{1}{8 \pi^{2}} \log (\Lambda / m)$. In fact, the obstruction spoils even the one-to-one correspondence between bare and renormalized parameters (at fixed cutoff) which must hold true in general and holds true also in the 1LRG improved relation (3.9).

Secondly, and even more seriously, one may verify that the above renormalization procedure does not remove all the logarithmic UV-cutoff dependence from the potential. That is, $V_{\text {eff }}(\phi)$ does not parametrically depend solely on $m^{2}$ and $\lambda$, but also on $\lambda_{0}$ and therefore on $\log \Lambda$. In a QFT with a Landau pole or obstruction, where the UV cutoff cannot be completely removed, we should accept at most an inverse power dependence of physical quantities on $\Lambda$.

To show this, consider the finite part of $I_{E}^{(1)}$, that is

$$
J\left(y-m^{2}\right)=I_{E}^{(1)}(y, \Lambda)-I_{E}^{(1)}\left(m^{2}, \Lambda\right)-I_{E}^{(2)}\left(m^{2}, \Lambda\right)\left(m^{2}-y\right)
$$

for any real $y$. By the use of $J$ Eq. (3.5) may be cast in the form

$$
\hat{V}^{\prime}(\xi)=\frac{1}{2} m^{2}+\frac{1}{12} \lambda \xi+\frac{1}{12}\left(\lambda+2 \lambda_{0}\right) J\left(2 \hat{V}^{\prime}(\xi)+\frac{1}{3} \lambda_{0} \xi-m^{2}\right) .
$$

This can be written more compactly

$$
\Delta \hat{V}^{\prime}(\xi)=\frac{1}{4} g J\left(2 \Delta \hat{V}^{\prime}(\xi)+\frac{1}{2} g \xi\right)
$$

with the definitions

$$
g=\frac{1}{3}\left(\lambda+2 \lambda_{0}\right), \quad \Delta \hat{V}^{\prime}(\xi)=\hat{V}^{\prime}(\xi)-\hat{V}^{\prime}(0)-\hat{V}^{\prime \prime}(0) \xi .
$$

It is quite clear now that the higher order derivatives (or vertex functions with vanishing incoming momenta) of $V_{\text {eff }}$, which are all contained in $\Delta \hat{V}^{\prime}$, depend explicitly on logarithms of the cutoff through the effective coupling $g$.

This problem was dealt with in $[8,24]$ by a direct renormalization of the HF gap equation, by setting

$$
\begin{aligned}
M^{2} & =m_{0}^{2}+\frac{1}{2} \lambda_{0} \xi+\frac{1}{2} \lambda_{0} I_{E}^{(1)}\left(M^{2}, \Lambda\right) \\
& =m^{2}+\frac{1}{2} \lambda \xi+\frac{1}{2} \lambda J\left(M^{2}-m^{2}\right)
\end{aligned}
$$

so that the bare-to-renormalized relations are found to be

$$
\lambda_{0}=\frac{\lambda}{1-\frac{1}{2} \lambda I_{E}^{(2)}\left(m^{2}, \Lambda\right)}, \quad m_{0}^{2}=m^{2}-\frac{1}{2} \lambda_{0} I_{E}^{(1)}\left(m^{2}, \Lambda\right)
$$

This procedure makes $M^{2}(\xi)$ and all higher derivatives of $\hat{V}(\xi)$ cutoff independent by definition, but fails in two respects. First of all the 1-loop beta function readout from Eq. (3.12) is 3 times smaller than the correct one [see Eq. (3.9)], which implies a mismatch already to first order in perturbation theory. Moreover the parametrizations (3.12) do not eliminate completely the cutoff logarithms, which remain in the quartic term of $V_{\text {eff }}(\phi)$ since the background field equation reads

$$
V_{\mathrm{eff}}^{\prime}(\phi)=\left[\frac{1}{2} M^{2}\left(\phi^{2}\right)-\frac{1}{6} \lambda_{0} \phi^{2}\right] \phi=0
$$

One could adjust the mismatch with the beta function by redefining the bare quantities appearing in the HF gap equation. If we make the $a d$ hoc replacements

$$
\begin{aligned}
& \lambda_{0} \rightarrow \tilde{\lambda}_{0}=\frac{\lambda_{0}}{1+\lambda_{0} I_{E}^{(2)}\left(m^{2}, \Lambda\right)} \\
& m_{0}^{2} \rightarrow \tilde{m}_{0}^{2}=m^{2}-\frac{1}{2} \tilde{\lambda}_{0} I_{E}^{(1)}\left(m^{2}, \Lambda\right)
\end{aligned}
$$

then the correct 1LRG relation (3.9) is recovered. As for the problem with the quartic term in $V_{\text {eff }}(\phi)$, a reasonable argument was put forward in [8] to justify the substitution $\tilde{\lambda}_{0} \rightarrow \lambda$ in Eq. (3.13).

We will see that this substitution, as well as the redefinition in Eq. (3.14), can be fully justified in the spirit of the 1LRG improvement of the HF approximation by an explicit diagrammatic analysis.

The problems just outlined have, in fact, a simple interpretation if we analyze the diagrams exactly resummed by the HF approximation. First, if we consider the renormalized mass, given by the two-legs vertex function with no momenta $\hat{V}^{\prime}(0)$, we see, by the first equation in (3.7), that it is dressed by daisy and superdaisy tadpole diagrams, as 


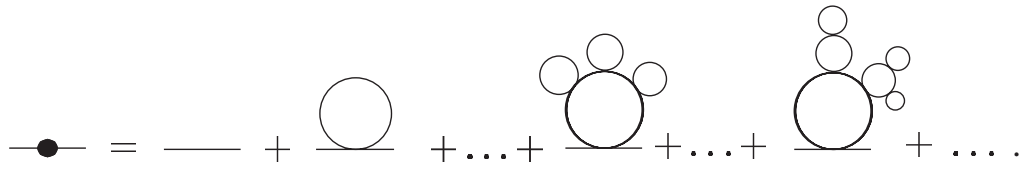

From now on we are going to work with this dressed mass and we will draw a simple solid line for the corresponding dressed propagator. Let us consider the quartic coupling; the second equation in (3.7) tells us it is obtained by "chain" diagrams (with dressed propagators).

$$
\gamma=X+X X+s y m+\searrow \bigcirc \times
$$

This is not the full diagrammatic expansion that corresponds to the 1LRG relation between $\lambda$ and $\lambda_{0}$ in Eq. (3.9). The latter in fact includes all local contributions with leading logs (LL) of the cutoff from all diagrams at every given loop order. So Eq. (3.16) misses the LL local parts of graphs like

$$
\ldots+\searrow(2+\operatorname{sym}+\ldots+>+s y m+\ldots
$$

This is the cause of the problems outlined above with the form Eq. (3.7) of the renormalized coupling $\lambda$.

Moreover, we can examine in the same spirit the diagrams contributing to higher order vertex functions (higher order derivatives of $\hat{V}$ in zero field). From Eq. (3.5) we see that they are convergent loop diagrams (loop with more than 2 propagators) with dressed propagators
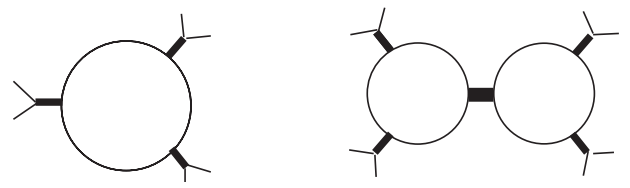

and vertices with the effective quartic coupling $g=$ $\left(2 \lambda_{0}+\lambda\right) / 3$

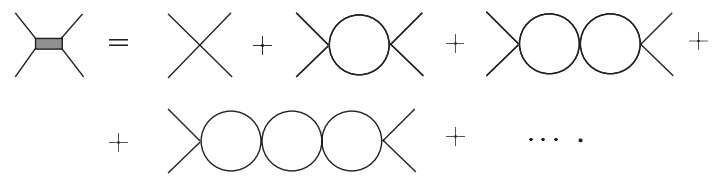

The reason of the presence of $g$ and not of the renormalized coupling $\lambda$ in higher order vertices is that the local LL contributions of diagrams like
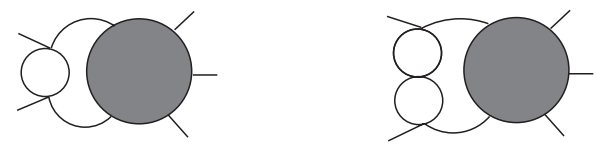

are not included in the resummation defined by the standard HF approximation as described by Eq. (3.10). Including such LL contributions would make the HF approximation renormalizable in terms of the reparametrizations of Eq. (3.7), that is with the pathological relation between $\lambda$ and $\lambda_{0}$. This is because the LL contributions of diagrams like

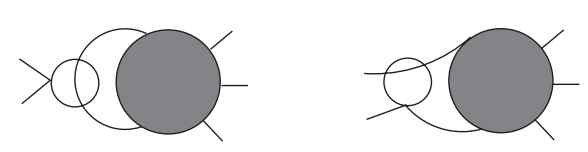

are still missing. Their proper inclusion would allow one to obtain everywhere the renormalized 1LRG improved coupling constant.

By these observations we are led to a natural way to modify the HF approximation in order to obtain a renormalized effective potential and the form (3.9) for the coupling constant that will ensure RG invariance in the general case, as we will see. The prescription is to include all missing contributions of the type (3.17) to $12 \hat{V}^{\prime \prime}(0)$ in order to obtain the 1LRG relation between $\lambda$ and $\lambda_{0}$ as in Eq. (3.9), and to include also the contributions of graphs of the form (3.20) and (3.21) in order to obtain $\lambda$ instead of $g$ in higher order vertex functions.

In conclusion we define a modified version of HF approximation by setting

$$
\begin{aligned}
2 \hat{V}^{\prime}(0) & =m^{2}=m_{0}^{2}+\frac{1}{2} \lambda_{0} I_{E}^{(1)}\left(m^{2}, \Lambda\right), \\
12 \hat{V}^{\prime \prime}(0) & =\lambda=\frac{\lambda_{0}}{1+\frac{3}{2} \lambda_{0} I_{E}^{(2)}\left(m^{2}, \Lambda\right)}, \\
\Delta \hat{V}^{\prime}(\xi) & =\frac{1}{4} \lambda J\left(2 \Delta \hat{V}^{\prime}(\xi)+\frac{1}{2} \lambda \xi\right) .
\end{aligned}
$$

This defines the renormalized potential $\hat{V}(\xi)$ and leads to the following equations:

$$
\begin{aligned}
M^{2} & =\frac{1}{2} \lambda \xi+m^{2}+\frac{1}{2} \lambda J\left(M^{2}-m^{2}\right), \\
0 & =\left(\frac{1}{2} M^{2}-\frac{1}{6} \lambda \phi^{2}\right) \phi,
\end{aligned}
$$

and these are finite and exactly in the form found in [8]. This gives a simple diagrammatic explanation of the substitution $\lambda_{0} \rightarrow \lambda$ made in [8] and of the statement (3.14). 


\section{B. The general case}

We are now in a position to deal with the general renormalization problem of the time-dependent and inhomogeneous HF approximation for $O(N)$-symmetric theory, as described by Eqs. (2.5) and (2.7).

Our first step is to derive a self-consistent functional equation for the effective action analogous to the one for the effective potential in Eq. (3.5). Let us introduce the squared mass parameter $m^{2}$. We choose $m^{2}$ to be the equilibrium mass (i.e. the physical mass of the unbroken symmetry phase), that is to say the solution of the mass-gap Eq. (3.3), generalized to $N>1$, in zero field.

$$
m^{2}=m_{0}^{2}+\frac{1}{6}(N+2) \lambda_{0} I_{E}^{(1)}\left(m^{2}, \Lambda\right) .
$$

Let us then define the free mode functions $u_{\boldsymbol{k} a}^{(0)}$ as solutions of

$$
\left(\square+m^{2}\right) u_{k a}^{(0)}(x)=0
$$

with the same initial conditions of the exact mode functions, that is

$$
u_{k a}^{(0)}(\boldsymbol{x}, 0)=u_{k a}(\boldsymbol{x}, 0), \quad \dot{u}_{\boldsymbol{k} a}^{(0)}(\boldsymbol{x}, 0)=\dot{u}_{\boldsymbol{k} a}(\boldsymbol{x}, 0) .
$$

We need also the free retarded and advanced Green functions

$$
\begin{aligned}
G_{R, i j}^{(0)}(x-y) & =\int \frac{d^{4} p}{(2 \pi)^{4}} \frac{\delta_{i j}}{p^{2}-m^{2}+i \epsilon p_{0}} e^{-i p(x-y)}, \\
G_{A, i j}^{(0)}(x-y) & =\int \frac{d^{4} p}{(2 \pi)^{4}} \frac{\delta_{i j}}{p^{2}-m^{2}-i \epsilon p_{0}} e^{-i p(x-y)}
\end{aligned}
$$

which allow one to cast Eq. (2.7) in the form of an integral equation (this is a quite standard way to proceed in the renormalization of out-of-equilibrium problems, see i.e. [35])

$$
u_{k a, i}(x)=u_{k a, i}^{(0)}(x)+\int d^{4} y G_{R i n}^{(0)}(x-y) \mathcal{V}_{n j}(y) u_{k a, j}(y),
$$

where

$$
\mathcal{V}_{i j}(x)=\frac{1}{3} \lambda_{0} \xi_{i j}(x)+2 \mathcal{F}^{\prime}[\xi]_{i j}(x)-m^{2} \delta_{i j}
$$

plays evidently the role of mean field for the mode functions. In these equations, and everywhere else from now on, all fields $\left(\phi\right.$, mode functions, $\mathcal{F}^{\prime}, \mathcal{V}$, etc. $)$ are to be thought of as defined only for positive times (initial conditions are at the limit point $t=0^{+}$) and all time integrations are restricted to positive values, as appropriate in an initial value problem.

In compact notation Eqs. (3.26) and (3.27) can be written as

$$
u_{k a}=u_{k a}^{(0)}+G_{R}^{(0)} \mathcal{V} u_{k a}, \quad \mathcal{V}=\frac{1}{3} \lambda_{0} \xi+2 \mathcal{F}^{\prime}-m^{2},
$$

where sums over internal $O(N)$ indices and integrations over space-time coordinates are understood. In particular, notice that $\mathcal{V}, \xi$, and $\mathcal{F}^{\prime}[\xi]$ act as multiplication operators over space-time.

The solution of Eq. (3.28) formally reads

$$
u_{k a}=\left[1-G_{R}^{(0)} \mathcal{V}\right]^{-1} u_{k a}^{(0)}
$$

so that the cutoffed correlation

$$
G_{i j}(x, y)=\operatorname{Re} \int_{|p|<\Lambda} \frac{d^{3} p}{(2 \pi)^{3}} u_{p a, i}(x) \bar{u}_{p a, j}(y)
$$

can be written

$$
G=G[\mathcal{V}]=\left[1-G_{R}^{(0)} \mathcal{V}\right]^{-1} G^{(0)}\left[1-\mathcal{V}^{T} G_{A}^{(0)}\right]^{-1},
$$

where

$$
G_{i j}^{(0)}(x-y)=\operatorname{Re} \int_{|p|<\Lambda} \frac{d^{3} p}{(2 \pi)^{3}} u_{p a, i}^{(0)}(x) \bar{u}_{p a, j}^{(0)}(y)
$$

is the free correlation function, which is entirely fixed by the initial conditions. In conclusion, by substituting the form (3.29) in Eq. (2.21), we obtain the sought selfconsistent equation for $\mathcal{F}^{\prime}$ as

$$
\begin{aligned}
\mathcal{F}^{\prime}[\xi]_{i j} & =\frac{1}{2}\left(m^{2} \delta_{i j}+\mathcal{V}[\xi]_{i j}\right)-\frac{1}{6} \lambda_{0} \xi_{i j}, \\
\mathcal{V}_{i j} & =\frac{1}{2} \lambda_{0} \tau_{i j k n}\left(\xi_{k n}+I[\mathcal{V}]_{k n}-I_{E}^{(1)} \delta_{k n}\right),
\end{aligned}
$$

where we have renamed the correlation at coincident points as

$$
I[\mathcal{V}]_{i j}(x)=G[\mathcal{V}]_{i j}(x, x) .
$$

Now, following closely the previous subsection, we may use these expressions, together with the general formula Eq. (2.22), to evaluate the renormalization conditions. To begin with, we consider the HF vacuum as an initial state for the quantum fluctuations. That is to say that we start from equilibrium initial conditions for the mode functions by choosing as $t=0$ kernels the solutions of the static problem of the previous subsection (generalization to the $N>1$ case is straightforward), that is

$$
\tilde{G}_{i j}(\boldsymbol{k})=\frac{\delta_{i j}}{2 \omega(\boldsymbol{k})}, \quad \omega(\boldsymbol{k})=\sqrt{\boldsymbol{k}^{2}+m^{2}}, \quad \tilde{S}_{i j}(\boldsymbol{k})=0 .
$$

Let us stress that, in spite of this choice, we are still considering an out-of-equilibrium problem since we allow for generic initial conditions for the background field. We will discuss the use of different initial kernels later on.

By the standard prescription we have to compute, using Eqs. (2.22) and (3.30) (the Fourier transform of), the twoand four-legs vertex functions $\Gamma^{(2)}$ and $\Gamma^{(4)}$ at some special values of the external momenta characterized by some given scale $s$. Owing to our assumption of unbroken $O(N)$ symmetry, we may choose to renormalize $\Gamma^{(2)}$ at 
zero momentum, while for $\Gamma^{(4)}$ we make the usual choice of the symmetric configuration. These special values of $\Gamma^{(2)}$ and $\Gamma^{(4)}$ will be identified, respectively, with the physical squared mass at equilibrium and with the coupling constant renormalized at the scale $s$.

Using the relations (2.6) and definitions (3.24) and (3.25) we obtain the following form for the free mode functions:

$$
u_{\boldsymbol{k} a, i}^{(0)}(x)=\frac{\delta_{i a}}{\sqrt{2 \omega(\boldsymbol{k})}} e^{i \boldsymbol{k} \cdot \boldsymbol{x}-i \omega(\boldsymbol{k}) t} .
$$

The two-legs function is obtained by calculating (3.30) in zero $\xi$ field. It is easy to check that $\mathcal{V}=0$ is a solution of Eq. (3.30) for $\xi=0$ if the mass renormalization Eq. (3.23) holds true. Using this fact and the general definition of vertex functions, Eq. (2.22), we obtain the free two-points vertex function

$$
\Gamma_{i j}^{(2)}(x \mid y)=\left(\square+m^{2}\right) \delta_{i j} \delta^{4}(x-y) .
$$

We recall that this is a vertex function in the physical representation of the CTP formalism, with one $\phi_{\Delta}$ and one $\phi_{c}$ insertion.

Now we have to calculate the four-legs function (one $\phi_{\Delta}$ and three $\phi_{c}$ ). From the variation of the second Eq. (3.30) with respect to $\xi$ at $\xi=0$ we find the integral equation

$$
\begin{aligned}
\vartheta_{i j k m}(x)= & \lambda_{0} \tau_{i j k m} \delta^{(4)}(x) \\
& -\frac{1}{2} \lambda_{0} \tau_{i j r s} \int d^{4} x^{\prime} I^{(2)}\left(x-x^{\prime}\right) \vartheta_{r s k m}\left(x^{\prime}\right)
\end{aligned}
$$

for the function

$$
\vartheta_{i j r s}\left(x-x^{\prime}\right)=\left.2 \frac{\delta \mathcal{V}[\xi]_{i j}(x)}{\delta \xi_{r s}\left(x^{\prime}\right)}\right|_{\xi=0},
$$

where

$$
I^{(2)}(x)=-\frac{2}{N} G_{R, i j}^{(0)}(x) G_{i j}^{(0)}(x)
$$

is the (suitably normalized) loop with one retarded and one correlation Green function. Equation (3.32) becomes algebraic in Fourier space

$$
\tilde{\vartheta}_{i j k m}(p)=\lambda_{0} \tau_{i j k m}-\frac{1}{2} \lambda_{0} \tilde{I}^{(2)}(p) \tau_{i j r s} \tilde{\vartheta}_{r s k m}(p)
$$

and is easily solved by

$$
\begin{aligned}
\tilde{\vartheta}_{i j k m}\left(p ; \lambda_{0}\right)= & \frac{1}{3} g_{1}\left(p ; \lambda_{0}\right)\left[\delta_{i k} \delta_{j m}+\delta_{j m} \delta_{i k}\right] \\
& +\frac{1}{3} g_{2}\left(p ; \lambda_{0}\right) \delta_{i j} \delta_{k m}
\end{aligned}
$$

with $g_{1}$ and $g_{2}$ defined as

$$
\begin{aligned}
& g_{1}\left(p ; \lambda_{0}\right)=\frac{\lambda_{0}}{1+\frac{1}{3} \lambda_{0} \tilde{I}^{(2)}(p)}, \\
& g_{2}\left(p ; \lambda_{0}\right)=\frac{g_{1}\left(p ; \lambda_{0}\right)}{1+\frac{1}{6}(N+2) \lambda_{0} \tilde{I}^{(2)}(p)} .
\end{aligned}
$$

We recall that, as appropriate to a causal initial value problem, all Fourier transforms are analytic in the upper complex $p_{0}$-half plane. Notice also that $\tilde{I}^{(2)}(p)=$ $\tilde{I}^{(2)}\left(p, m^{2}, \Lambda\right)$ depends on the UV-cutoff $\Lambda$ through the initial time correlation function $G_{i j}^{(0)}(x)$,

$$
\tilde{I}^{(2)}\left(p, m^{2}, \Lambda\right)=-\frac{2}{N} \int_{|k|<\Lambda} \frac{d^{4} k}{(2 \pi)^{4}} \tilde{G}_{R i j}^{(0)}(p-k) \tilde{G}_{i j}^{(0)}(k)
$$

so that, as long as $\Lambda$ is finite, it is a function of both the time component $p_{0}$ and of the Euclidean 3D length $|\boldsymbol{p}|$, rather than just of the Lorentz invariant $p^{2}=p_{0}^{2}-\boldsymbol{p}^{2}$.

We now apply the relation between $\mathcal{V}$ and $\mathcal{F}^{\prime}$ in (3.26) and the general definition (2.22) and obtain the following form of the four-legs vertex function:

$$
\begin{aligned}
\Gamma_{i \mid j k m}^{(4)}\left(x_{1} \mid x_{2}, x_{3}, x_{4}\right) & \\
= & \int\left[\prod_{n=1}^{3} \frac{d^{4} p_{n}}{(2 \pi)^{4}}\right] \hat{\Gamma}_{i \mid j k m}^{(4)}\left(p_{2}, p_{3}, p_{4}\right) e^{-i \sum_{n} p_{n} \cdot\left(x_{1}-x_{n}\right)}, \\
\tilde{\Gamma}_{i \mid j k m}^{(4)}= & -2 \lambda_{0} \tau_{i j k m}+\tilde{\vartheta}_{i j k m}\left(p_{3}+p_{4}\right)+\tilde{\vartheta}_{i k j m}\left(p_{2}+p_{4}\right) \\
& +\tilde{\vartheta}_{i m k j}\left(p_{2}+p_{3}\right) .
\end{aligned}
$$

Notice the structure of $\tilde{\Gamma}^{(4)}$ as a sum of the three terms, for the $s, t$, and $u$ channels, with the same functional form given by $\tilde{\vartheta}(p)-2 \lambda_{0} \tau / 3$. This structure is characteristic of the HF approximation, as can be inferred from the general formula (2.22).

In the present out-of-equilibrium context, we can define the symmetric point at the scale $s$ as

$$
p_{3}+p_{4}=p_{3}+p_{2}=p_{2}+p_{4}=q_{s} \equiv(0, \boldsymbol{q}), \quad|\boldsymbol{q}|=s
$$

with an arbitrary direction $\hat{\boldsymbol{q}}$. Evaluating $\tilde{\Gamma}^{(4)}$ at this point yields the renormalization condition

$$
\begin{aligned}
\lambda \tau_{i j k m} & =\left.\tilde{\Gamma}_{i \mid j k m}^{(4)}\right|_{\text {sym.pt } \mu} \longrightarrow \lambda \\
& =-2 \lambda_{0}+2 g_{1}\left(q_{s} ; \lambda_{0}\right)+g_{2}\left(q_{s} ; \lambda_{0}\right) .
\end{aligned}
$$

This result should be compared to the corresponding one in the static $N=1$ case, that is Eqs. (3.7) and (3.11). We see that, in the current context with generic $N$ and generic renormalization scale, the effective coupling $3 g$ is split into $2 g_{1}$ and $g_{2}$, both scale dependent.

Before going any further in discussing renormalizability and RG invariance (independence on the scale $s$ ), it is convenient to calculate a "quasirenormalized" form of the self-consistent equation (3.30) for $\mathcal{F}^{\prime}$ analogous to the Eq. (3.10) found for the effective potential.

First of all let us define $J[\mathcal{V}]$ as the part of the integral term $I[\mathcal{V}]$ in Eq. (3.30) that does not contain any divergent integral

$$
J(x)=I(x)-\left[G^{(0)}+G_{R}^{(0)} \mathcal{V} G^{(0)}+G^{(0)} \mathcal{V}^{T} G_{A}^{(0)}\right](x, x)
$$

that is, in Fourier space 


$$
\tilde{J}_{k m}(p)=\tilde{I}_{k m}(p)-I_{E}^{(1)} \delta^{(4)}(p) \delta_{k m}+\tilde{I}^{(2)}(p) \tilde{\mathcal{V}}_{k m}(p),
$$

where $\delta^{(4)}(p)$ stands for $-i\left(p_{0}+i \epsilon\right)^{-1} \delta^{(3)}(\boldsymbol{p})$, as appropriate in this out-of-equilibrium context.

The second of Eqs. (3.30) can now be rewritten as

$$
\tilde{V}_{i j}(p)=\frac{1}{2} \tilde{\vartheta}_{i j k m}\left(p ; \lambda_{0}\right)\left\{\tilde{\xi}_{k m}(p)+\tilde{J}[\mathcal{V}]_{k m}(p)\right\} .
$$

Now, we can define

$$
\begin{aligned}
\Delta \tilde{\mathcal{F}}_{i j}^{\prime}(p)= & \tilde{\mathcal{F}}_{i j}^{\prime}(p)-\frac{1}{2} m^{2} \delta_{i j} \delta^{(4)}(p)-\left[\frac{1}{4} \tilde{\vartheta}_{i j k m}(p)\right. \\
& \left.-\frac{1}{6} \lambda_{0} \delta_{i k} \delta_{j m}\right] \tilde{\xi}_{k m}(p)
\end{aligned}
$$

and, by substituting in (3.40), we obtain the HF selfconsistency equation in the quasirenormalized form that we were looking for, that is

$$
\begin{gathered}
\Delta \tilde{\mathcal{F}}_{i j}^{\prime}(p)=\frac{1}{4} \tilde{\vartheta}_{i j k m}(p) \tilde{J}[\mathcal{V}]_{k m}(p), \\
\tilde{V}_{i j}(p)=2 \Delta \tilde{\mathcal{F}}_{i j}^{\prime}(p)+\frac{1}{2} \tilde{\vartheta}_{i j k m}(p) \tilde{\xi}_{k m}(p)
\end{gathered}
$$

which is to be compared with Eq. (3.10).
We can now repeat the analysis of the previous subsection in this more general case. As we will see, conclusions will be rather similar.

First of all, exactly as it happened in the static case, the renormalization of the mass parameter receives contributions from daisy and superdaisy tadpoles with the topologies shown in (3.15). These contributions are actually not affected by momenta entering in the two-legs function and simply define the bare mass in terms of the renormalized one according to Eq. (3.23). From now on we deal with dressed propagators, i.e. propagators with the renormalized mass.

We look then at the coupling renormalization in Eq. (3.38). It exhibits the same pathological behavior of the static $N=1$ case, Eq. (3.7), now with a $N$-dependent Landau obstruction. Most importantly, from the explicit form of the HF four-legs function in Eq. (3.37) we see that imposing a finite value to $\lambda$ at a certain chosen scale $s$ fails to render finite the four-legs function with generic incoming momenta and, in particular, the coupling $\lambda^{\prime}$ at any other scale $s^{\prime} \neq s$. We can correct both shortcomings with the same strategy of the static $N=1$ case. In fact, the diagrammatic analysis of the HF four-legs function shows that it is given by the resummation of "bubble" graphs

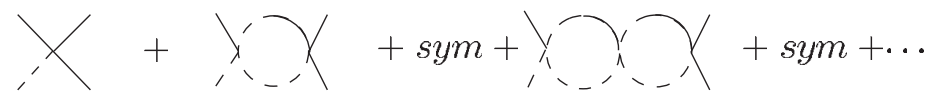

where we recall that dotted (solid) lines represent $\phi_{\Delta}\left(\phi_{c}\right)$ propagators and external legs coupled with the vertices (2.19) of the physical representation. This resummation misses all leading logarithmically divergent contributions coming from nonbubble diagrams. In the usual in-out formalism their inclusion provides the standard 1LRG relation

$$
\frac{1}{\lambda} \simeq \frac{1}{\lambda}+\beta_{0} \lambda \log \Lambda+\cdots,
$$

where $\beta_{0}=(N+8) / 48 \pi^{2}$ is the first coefficient of the beta function and the dots stand for the scheme-dependent finite parts. Now the HF approximation provides a precise scheme where leading logarithms and finite parts are all contained in the 1-loop bubble $\tilde{I}^{(2)}\left(q_{s}, m^{2}, \Lambda\right)$. Hence we fix the finite parts in the standard 1LRG relation above, by choosing the complete parametrization

$$
\left.\lambda_{0}(\lambda, s / m, \Lambda / m)\right|_{1 \mathrm{LRG}}=\frac{\lambda}{1-\frac{N+8}{6} \lambda \tilde{I}^{(2)}\left(q_{s}, m^{2}, \Lambda\right)}
$$

which generalizes to the present nonstatic case with generic $N$ the static $N=1$ result in Eq. (3.17) and replaces the pure HF parametrization of Eq. (3.38). Notice that in the out-of-equilibrium formalism there could be a priori a complication with nonlocal finite parts, since the four-legs function $\tilde{\Gamma}^{(4)}$, even if evaluated at the symmetric point, need not be in principle completely symmetric in its four $O(N)$ indices (recall that it has one $\phi_{\Delta}$ leg and three $\phi_{c}$ legs). However the symmetry in the three $\phi_{c}$ legs is enough to fix the $\tau$ tensor as the unique $O(N)$-invariant fourth-rank tensor, so that a unique coupling constant renormalization can occur, including finite parts, just like in the standard inout formalism.

Let us now consider the four-legs function $\tilde{\Gamma}^{(4)}$ at generic values of the external momenta. We need to modify its HF form (3.37) in such a way to meet the following requirements:

(i) It must be the sum of the three separated channels $s$, $t$, and $u$ with the same functional form in each channel, as dictated by the mean-field form of the equations of motion.

(ii) It must reduce to the relation $\lambda=$ $\lambda\left(\lambda_{0}, s / m, \Lambda / m\right)$, the inverse of Eq. (3.44), at the normalization point.

(iii) It must be free of cutoff logarithms for any value of the external momenta.

(iv) It must match the perturbative one-loop result and respect $O(N)$ symmetry.

A specific form meeting all requirements is given by

$$
\begin{aligned}
\tilde{\Gamma}_{i \mid j k m}^{(4)}\left(p_{2}, p_{3}, p_{4}\right)= & \lambda_{r}\left(p_{3}+p_{4}\right) \ell_{i k j m}+\lambda_{r}\left(p_{2}+p_{4}\right) \\
& \times \ell_{i j k m}+\lambda_{r}\left(p_{3}+p_{2}\right) \ell_{i m j k},
\end{aligned}
$$


where the tensor $\ell$ reads

$$
\ell_{i j k m}=\frac{1}{3(N+8)}\left[(N+4) \delta_{i j} \delta_{k m}+2\left(\delta_{i k} \delta_{j m}+\delta_{i m} \delta_{j k}\right)\right]
$$

and $\lambda_{r}(p)$ is the running coupling constant, connected to coupling $\lambda=\lambda_{r}\left(q_{s}\right)$ at the scale $s$ by the relation

$$
\frac{1}{\lambda_{r}(p)}=\frac{1}{\lambda}+\frac{N+8}{6}\left[\tilde{I}^{(2)}(p)-\tilde{I}^{(2)}\left(q_{s}\right)\right]
$$

that involves by construction only a subtracted integral, so that one may now take the limit $\Lambda \rightarrow \infty$. Of course, this defines a meaningful $\lambda_{r}(p)$ only for $p$ timelike or $p$ spacelike but such that $-p^{2}$ is smaller than the Landau pole.

It should be noted that Eq. (3.45) is not the unique possibility, given the requirements listed above, since it could be modified at low momenta by allowing a weak dependence on the normalization scale $s$. Here we choose to fulfill exactly the one-loop RG invariance, which fixes completely Eq. (3.45). In this way we are modifying the HF approximation in such a way to include the correct leading logarithm contributions due to the missing CTP diagrams with the topology in Eq. (3.17). At the same time we are approximating the whole momentum behavior of these diagrams according to the one-loop RG.

We still have to consider the higher order vertex functions. These are encoded by construction in the functional $\Delta \tilde{\mathcal{F}}_{i j}^{\prime}$ defined in (3.41). From the HF self-consistency equation (3.42) for $\Delta \tilde{\mathcal{F}}_{i j}^{\prime}$ we can see that an explicit dependence on the logarithm of the cutoff is still present even after the coupling constant renormalization. The reason is in the presence of the effective vertex $\vartheta_{i j k m}(p)$ in Eq. (3.40), which corresponds to CTP diagrams of the same type of those drawn in (3.19). Exactly in the same way of the $N=1$ static case [see Eqs. (3.18) and (3.19)] we have to include other contributions containing the correct leading logarithms that come from the $\phi_{\Delta}-\phi_{c}$ diagrams corresponding to those in Eqs. (3.20) and (3.21). In order to obtain renormalizability and maintain the single channel structure of the effective vertex (which is a direct consequence of the mean-field approximation), these contributions have to be taken momentum dependent in such a way as to determine a leading log structure for the effective vertex in place of $\vartheta_{i j k m}(p)$. Then finiteness, agreement with the lowest perturbative order, and one-loop RG invariance at all momentum scales require the resulting structure to be simply $\lambda_{r}(p) \tau_{i j k m}$.

In conclusion, we modify Eq. (3.42) in the following way:

$$
\begin{aligned}
\tilde{\mathcal{F}}_{i j}^{\prime}(p) & =\Delta \tilde{\mathcal{F}}_{i j}^{\prime}(p)+\frac{1}{2} m^{2} \delta_{i j} \delta^{(4)}(p)+\frac{1}{4} \lambda_{r}(p) \ell_{i j k m} \tilde{\xi}_{k m}(p), \\
\Delta \tilde{\mathcal{F}}_{i j}^{\prime}(p) & =\frac{1}{4} \tilde{\lambda}_{r}(p) \tau_{i j k m} \tilde{J}[\mathcal{V}]_{k m}(p), \\
\tilde{V}_{i j}(p) & =2 \Delta \tilde{\mathcal{F}}_{i j}^{\prime}(p)+\frac{1}{2} \lambda_{r}(p) \tau_{i j k m} \tilde{\xi}_{k m}(p) .
\end{aligned}
$$

The renormalized equations of motion are then

$$
\begin{aligned}
& {\left[\left(\square+m^{2}\right) \delta_{i j}+\mathcal{V}_{i j}(x)-\frac{1}{3} \Xi_{i j}(x)\right] \phi_{j}(x)=0,} \\
& {\left[\left(\square+m^{2}\right) \delta_{i j}+\mathcal{V}_{i j}(x)\right] u_{k a, j}(x)=0,} \\
& \tilde{\mathcal{V}}_{i j}(p)=\frac{1}{2} \lambda_{r}(p) \tau_{i j k m}\left[\tilde{\xi}_{k m}(p)+\tilde{I}_{k m}(p)\right. \\
& \left.\quad-I_{E}^{(1)} \delta^{(4)}(p) \delta_{k m}+\tilde{I}^{(2)}(p) \tilde{\mathcal{V}}_{k m}(p)\right],
\end{aligned}
$$

where $\Xi_{i j}(x)$ is the inverse Fourier transform of $\tilde{\Xi}_{i j}(p)=$ ${ }_{2}^{3} \lambda_{r}(p)\left(\tau_{i j k m}-\ell_{i j k m}\right) \tilde{\xi}_{k m}(p)$ and we recall that $\xi(x)=$ $\phi_{i}(x) \phi_{j}(x)$ and $\tilde{I}_{k m}(p)$ is the Fourier transform of

$$
I[\mathcal{V}]_{i j}(x)=\operatorname{Re} \int_{|p|<\Lambda} \frac{d^{3} p}{(2 \pi)^{3}} u_{p b, i}(x) \bar{u}_{p b, j}(x)
$$

which is a functional of the mean-field $\mathcal{V}$ through the evolution equation for the mode functions.

The absence of any divergent cutoff dependence in the modified HF approximation defined by Eqs. (3.47) or by the equations of motion (3.48) is manifest, since they involve only the finite running coupling constant and the fully subtracted (Fourier transform of the) equal point correlation $I_{i j}(x)$. Comparing the expression for $\tilde{V}_{i j}(p)$ with the original one in Eq. (3.30), that reads in Fourier space,

$$
\tilde{V}_{i j}(p)=\frac{1}{2} \lambda_{0} \tau_{i j k n}\left[\tilde{\xi}_{k n}(p)+\tilde{I}_{k n}(p)-I_{E}^{(1)} \delta^{(4)}(p) \delta_{k n}\right]
$$

we see that our improved renormalization simply amounts to performing the logarithmic subtraction on $I[\mathcal{V}]_{i j}$ while promoting the bare coupling $\lambda_{0}$ to the running one $\lambda_{r}(p)$. As a consequence, the $p$ dependence of $\lambda_{r}(p)$ and $\tilde{I}^{(2)}(p)$ imply a space-time nonlocality of the modified HF selfconsistency equation for the mean-field $\mathcal{V}(x)$, which now depends on the whole history of the background $\phi$ and of the mode functions $u_{k a}$ from time zero to time $t$. A similar space-time nonlocality appears in the equation of motion for the background field $\phi$. Causality in this nonlocal evolution is guaranteed by analyticity in the upper $p_{0}$-half plane of $\tilde{I}^{(2)}(p)$. Let us observe, however, that the third equation in Eqs. (3.48) does not provide an explicit expression of the mean-field $\mathcal{V}$ in terms of the background and of the mode functions. To obtain such an expression, we must solve for $\mathcal{V}$, paying the price of losing manifest finiteness. In practice, this reduces to the simple matrix inversion already performed on Eq. (3.32) and yields

$$
\begin{aligned}
\tilde{\mathcal{V}}_{i j}(p)= & -\frac{1}{2} \tilde{\vartheta}_{i j k m}\left(p ;-\lambda_{r}(p)\right)\left[\tilde{\xi}_{k m}(p)+\tilde{I}_{k m}(p)\right. \\
& \left.-I_{E}^{(1)} \delta^{(4)}(p) \delta_{k m}\right],
\end{aligned}
$$

where the $\vartheta_{i j k m}(p ; u)$ is the function defined in Eq. (3.34). In coordinate space this becomes

$$
\begin{aligned}
\mathcal{V}_{i j}(x)= & \frac{1}{3} \int d^{4} x^{\prime}\left\{2 \gamma _ { 1 } ( x - x ^ { \prime } ) \left[\phi_{i}\left(x^{\prime}\right) \phi_{j}\left(x^{\prime}\right)+I_{i j}\left(x^{\prime}\right)\right.\right. \\
& \left.-\delta_{i j} I_{E}^{(1)}\right]+\delta_{i j} \gamma_{2}\left(x-x^{\prime}\right)\left[\phi^{2}\left(x^{\prime}\right)\right. \\
& \left.\left.+I_{k k}\left(x^{\prime}\right)-N I_{E}^{(1)}\right]\right\}
\end{aligned}
$$


where $\gamma_{1}(x)$ and $\gamma_{2}(x)$ are the inverse Fourier transforms of the functions $-g_{1}\left(p ;-\lambda_{r}(p)\right)$ and $-g_{2}\left(p ;-\lambda_{r}(p)\right)$, respectively, which can be read from Eq. (3.35) and are indeed analytic in the upper $p_{0}$-half plane. For instance, we have explicitly

$$
\begin{aligned}
\gamma_{1}(x)= & \int \frac{d^{4} p}{(2 \pi)^{4}} \\
& \times \frac{\lambda e^{-i p \cdot x}}{1+\frac{1}{6} \lambda\left[(N+1) \tilde{I}^{(2)}(p)-\left(N+\frac{4}{3}\right) \tilde{I}^{(2)}\left(q_{s}\right)\right]}
\end{aligned}
$$

with an even more involved expression for $\gamma_{2}(x)$. Clearly $\gamma_{1,2}$ depend logarithmically on the cutoff $\Lambda$ through an incompletely subtracted $\tilde{I}^{(2)}(p)$, but, by construction, this logarithms cancels out with those coming from the incomplete subtraction $I_{i j}(x)-\delta_{i j} I_{E}^{(1)}$ on the equal point correlation of the mode functions. The memory functions $\gamma_{1}(x)$ and $\gamma_{2}(x)$, as well as the inverse Fourier transform of $\lambda_{r}(p)$ which enters in the evolution equation of the background field [see Eq. (3.48)], all share the same behavior for large timelike values of $x$, namely, a $\left(x^{2}\right)^{-1 / 2}$ decay modulated by oscillating factors.

When $N=1$ things simplify considerably and we find for the mean field

$$
\begin{aligned}
\mathcal{V}(x)= & \int d^{4} x^{\prime} \gamma\left(x-x^{\prime}\right)\left\{\phi^{2}\left(x^{\prime}\right)+\int \frac{d^{3} k}{(2 \pi)^{3}}\left[\left|u_{\boldsymbol{k}}(x)\right|^{2}\right.\right. \\
& \left.\left.-\frac{1}{2 \omega(\boldsymbol{k})}\right]\right\},
\end{aligned}
$$

where

$$
\begin{aligned}
\gamma(x) & =\frac{2}{3} \gamma_{1}(x)+\frac{1}{3} \gamma_{2}(x) \\
& =\frac{1}{2} \int \frac{d^{4} p}{(2 \pi)^{4}} \frac{\lambda e^{-i p \cdot x}}{1+\lambda\left[I^{(2)}(p)-\frac{3}{2} I^{(2)}\left(q_{s}\right)\right]}
\end{aligned}
$$

while mode functions and background evolve according to

$$
\begin{aligned}
{\left[\square+m^{2}+\mathcal{V}(x)\right] u_{k}(x) } & =0, \\
{\left[\square+m^{2}+\mathcal{V}(x)-\frac{1}{3} \int d^{4} x^{\prime} \gamma_{r}\left(x-x^{\prime}\right) \phi^{2}\left(x^{\prime}\right)\right] \phi(x) } & =0,
\end{aligned}
$$

where

$$
\gamma_{r}(x)=\int \frac{d^{4} p}{(2 \pi)^{4}} \frac{\lambda e^{-i p \cdot x}}{1+\frac{3}{2} \lambda\left[I^{(2)}(p)-I^{(2)}\left(q_{s}\right)\right]}
$$

is the ultraviolet finite inverse Fourier transform of the running coupling constant $\lambda_{r}(p)$.

On the opposite end, when $N \rightarrow \infty$, one can verify that the standard local evolution equations [3] are recovered from Eqs. (3.48). To this end, it is convenient to restrict first to the special case of a background field which maintains a fixed direction, that is $\phi_{i}(x)=\phi(x) v_{i}$, with $v$ some fixed $N$-dimensional unit vector. In fact in this case we can reduce Eqs. (3.48) to an index-free form by introducing longitudinal and transversal projectors

$$
P_{L, i j}=v_{i} v_{j}, \quad P_{T, i j}=\delta_{i j}-P_{L, i j} .
$$

Then we can set

$$
\begin{aligned}
& u_{k a, i}(x)=u_{k L}(x) P_{L, i a}+u_{k T}(x) P_{T, i a}, \\
& \mathcal{V}_{i j}(x)=\mathcal{V}_{L}(x) P_{L, i j}+\mathcal{V}_{T}(x) P_{T, i j}
\end{aligned}
$$

which are compatible with the initial condition in Eq. (3.31) if we assume that the longitudinal and transverse parts of the mode functions and their derivatives are equal at the initial time. By substituting into the equations of motion and projecting one obtains

$$
\begin{aligned}
& {\left[\square+m^{2}+\mathcal{V}_{T}(x)\right] u_{k T}(x)=0,} \\
& {\left[\square+m^{2}+\mathcal{V}_{L}(x)\right] u_{k L}(x)=0,} \\
& {\left[\square+m^{2}+\tilde{V}_{L}(x)-\frac{1}{3} \Phi(x)\right] \phi(x)=0,} \\
& \tilde{V}_{L}(p)=\frac{1}{2} \tilde{\Phi}(p)+\frac{1}{6} \lambda_{r}(p)\left[(N-1) \tilde{J}_{T}(p)+3 \tilde{J}_{L}(p)\right], \\
& \tilde{\mathcal{V}}_{T}(p)=\frac{1}{6} \tilde{\Phi}(p)+\frac{1}{6} \lambda_{r}(p)\left[(N+1) \tilde{J}_{T}(p)+\tilde{J}_{L}(p)\right],
\end{aligned}
$$

where $\Phi(x)$ is the inverse Fourier transform of $\tilde{\Phi}(p)=$ $\lambda_{r}(p) \tilde{\xi}(p)$ [recall that $\xi(x)=\phi^{2}(x)$ ] and the meaning of $J_{L}$ and $J_{T}$ is obvious [see Eq. (3.39)]. Again, the formulation in momentum space for the mean-field $\mathcal{V}$, while convenient to avoid lengthy convolution integrals and to allow for an easy check of finiteness, does not make causality manifest. The opposite situation, with finiteness hidden and causality manifest would follow by solving explicitly for $\mathcal{V}$ and then reverting to coordinate space as done above for the general case [see Eq. (3.49)] or for the $N=1$ case.

Now let us rescale the coupling and the background field as prescribed by the standard large $N$ procedure

$$
\lambda \rightarrow \lambda / N \quad \phi(x) \rightarrow \sqrt{N} \phi(x) .
$$

By substituting in (3.50) and taking the limit on $N$ we obtain (notice that the longitudinal mode functions decouple)

$$
\begin{aligned}
{\left[\square+m^{2}\right.} & \left.+\mathcal{V}_{T}(x)\right] u_{k T}(x)=0, \\
{\left[\square+m^{2}\right.} & \left.+\mathcal{V}_{T}(x)\right] \phi(x)=0, \\
\mathcal{V}_{T}(x)= & \frac{1}{6} \lambda\left\{\phi^{2}(x)+\int \frac{d^{3} k}{(2 \pi)^{3}}\left[\left|u_{\boldsymbol{k}}(x)\right|^{2}-\frac{1}{2 \omega(\boldsymbol{k})}\right]\right. \\
& \left.+I^{(2)}(s) \mathcal{V}_{T}(x)\right\}
\end{aligned}
$$

which are the usual, local, and renormalized large $N$ evolution equations. We remark that $\lambda$ is the renormalized coupling at the scale $s$ while the nonlocal terms [with the running coupling $\lambda(p)]$ have all disappeared by explicit cancellation. This is indeed what was to be expected, on the basis of the same diagrammatic analysis that leads to our improvement of the HF approximation, since the non- 
renormalizable terms in the standard $\mathrm{HF}$ approach, as well as the contribution we have added to cure the problem, all come from diagrams that are at least $1 / N$ suppressed.

We conclude this lengthy subsection with an explicit check of the renormalization-group invariance of our improved HF approximation, by verifying that indeed all vertex functions obtained by Eqs. (3.47) are solutions of the Callan-Symanzik (CS) equation. These equations state the RG invariance of any observable $\mathcal{O}$ which is a function of coordinates $x_{i}$ (or momenta $p_{i}$ ), of the scale $s$, the coupling $\lambda$, and the parameter $\sigma=m^{2} / s^{2}$, that is

$\left[s \frac{\partial}{\partial s}+\beta(\lambda, \sigma) \frac{\partial}{\partial \lambda}+\beta_{\phi^{2}}(\lambda, \sigma) \frac{\partial}{\partial \sigma}\right] \mathcal{O}\left(\left\{x_{i}\right\} \mid s, \lambda, \sigma\right)=0$

with $\beta$ and $\beta_{\phi^{2}}$ functions to be determined. Notice the absence of the term with the anomalous dimension due to the lack of field renormalization in HF approximation. By applying Eq. (3.51) to the two-legs function one obtains $\beta_{\phi^{2}}=-2 \sigma$. Then by applying to the four-legs function one obtains

$$
\beta(\lambda, \sigma)=-\left.\frac{1}{6}(N+8) \lambda^{2} \lim _{\Lambda \rightarrow \infty} s \frac{\partial}{\partial s} I^{(2)}\left(s, m^{2}, \Lambda\right)\right|_{m^{2}=\sigma s^{2}}
$$

Then for $s^{2} \gg m^{2}$ we have $\beta \simeq(N+8) / 48 \pi^{2} \lambda^{2}$ as expected. Now, is easy to check that all free propagators with dressed mass satisfy the CS equation since they are functions of $m^{2}$ alone. Then, since the generic $n$-legs function is a functional of the propagators and of the RG-invariant running coupling $\lambda_{r}(p)$ through the four-legs function, one immediately concludes that it satisfies Eq. (3.51).

\section{Other initial states and nonzero temperatures}

As stated in the previous subsection, with generic initial conditions on the background field Eqs. (3.48) already describe an out-of-equilibrium problem, in spite of the choice in Eq. (3.31) of equilibrium initial conditions on the quantum fluctuations. It is nonetheless sensible to ask whether and how we can choose different initial conditions for the mode functions without spoiling the properties of renormalizability and RG invariance.

What might happen can be shown by the following example. Let us consider, in the simple $N=1$ case, an initial state of the same form of the HF vacuum but with a different mass $M$, which could be for instance the solution of the gap Eq. (3.3) in the case of a uniform background. This is a frequent choice in dealing with nonequilibrium problems and has a precise physical meaning, since it corresponds to the minimization of the quantum fluctuation part of the HF energy at a fixed uniform background. At any rate, as soon as we assume as initial conditions

$$
\begin{gathered}
\tilde{\mathcal{G}}_{i j}(\boldsymbol{k})=\frac{\delta_{i j}}{2 \Omega(\boldsymbol{k})}, \quad \Omega(\boldsymbol{k})=\sqrt{\boldsymbol{k}^{2}+M^{2}}, \\
\tilde{S}_{i j}(\boldsymbol{k})=0
\end{gathered}
$$

with $M \neq m$, then the free mode functions have more than one frequency component

$$
\begin{aligned}
u_{\boldsymbol{k}}^{(0)}(x)= & \frac{e^{i \boldsymbol{k} \cdot \boldsymbol{x}}}{2 \sqrt{2 \Omega(\boldsymbol{k})}}\left\{\left[1+\frac{\Omega(\boldsymbol{k})}{\omega(\boldsymbol{k})}\right] e^{-i \omega(\boldsymbol{k}) t}\right. \\
& \left.+\left[1-\frac{\Omega(\boldsymbol{k})}{\omega(\boldsymbol{k})}\right] e^{-i \omega(\boldsymbol{k}) t}\right\}
\end{aligned}
$$

and the free correlation function is no longer translationally invariant in time. Then the integral term in Eqs. (3.48) (recall that here $N=1$ )

$$
\tilde{I}(p)-I_{E}^{(1)} \delta^{(4)}(p)+I^{(2)}(p) \tilde{V}(p)
$$

in spite of the subtractions still contains the superficially divergent contribution

$$
\int \frac{d^{3} k}{(2 \pi)^{3}} \frac{m^{2}-M^{2}}{4 \omega(\boldsymbol{k})^{3}} \cos 2 \omega(\boldsymbol{k}) t
$$

that indeed diverges with the cutoff when $t=0$. These initial time singularities have first been discussed in [36] and removed by a Bogoliubov transformation on the initial state, which in practice amounts to a redefinition of the initial kernel in such a way that the leading terms of a highmomentum expansion are the same as at equilibrium

$$
\hat{\mathcal{G}}(\boldsymbol{k}) \sim \frac{1}{2 \sqrt{\boldsymbol{k}^{2}}}+\frac{m^{2}}{4\left(\boldsymbol{k}^{2}\right)^{3 / 2}}+\cdots .
$$

In fact, one may verify that the initial singularity (and any other divergence as well) is absent for any choice of kernel having the above large $\boldsymbol{k}$ expansion. A simple interpretation is that the renormalization procedure ensures finiteness for any initial Gaussian state belonging to the same Fock space of the HF vacuum. From this simple example we can extrapolate the generic condition on the shortdistance behavior of the initial state kernel

$$
\hat{\mathcal{G}}(\boldsymbol{x}, \boldsymbol{y}) \simeq \frac{1}{4 \pi^{2}|\boldsymbol{x}-\boldsymbol{y}|}+\frac{m^{2}}{8 \pi^{2}} \log |\boldsymbol{x}-\boldsymbol{y}|+\cdots
$$

that ensures the cancellation of all divergent terms is guaranteed by mass and coupling constant renormalization. This conclusion can be immediately extended to the generic $N>1$ case.

Before concluding let us say some words about the case when the initial state has a nonzero temperature $T$. The formalism for pure state dynamics introduced in Sec. II B can be easily generalized to statistical mixtures defined by Gaussian density matrices, 


$$
\begin{aligned}
\rho\left[\varphi_{1}, \varphi_{2}\right]= & \mathcal{N} \exp \left\{i\left\langle p \mid \varphi_{1}-\varphi_{2}\right\rangle\right. \\
& -\left\langle\Delta \varphi_{1}\left|\left[\frac{1}{4} \mathcal{G}^{-1}+i S\right]\right| \Delta \varphi_{1}\right\rangle \\
& -\left\langle\Delta \varphi_{2}\left|\left[\frac{1}{4} \mathcal{G}^{-1}-i S\right]\right| \Delta \varphi_{2}\right\rangle \\
& \left.+\frac{1}{2}\left\langle\Delta \varphi_{1}\left|\mathcal{G}^{-1 / 2} \zeta \mathcal{G}^{-1 / 2}\right| \Delta \varphi_{2}\right\rangle\right\},
\end{aligned}
$$

where $\Delta \varphi_{1} \equiv \varphi_{i}-\phi$. The parameters are the background field $\phi$ and its momentum $p$, the symmetric kernels $G, S, \gamma$ and the antisymmetric $\sigma$ (with $\zeta=\gamma+4 i G^{1 / 2} \sigma G^{1 / 2}$ ). The normalizability of the state $(\operatorname{Tr}[\rho]=1)$ requires $\mathcal{G}^{-1 / 2}(1-\gamma) \mathcal{G}^{-1 / 2}$ to be positive. In CTP formalism the generalization proceeds simply by substituting $\Psi\left[\varphi_{+}\right] \bar{\Psi}\left[\varphi_{-}\right]$with $\rho\left[\varphi_{+}, \varphi_{-}\right]$in the path integral of Eq. (2.10). Then HF equations are still as before while more general initial conditions for the mode functions are allowed. Let us consider an initial state with translationally invariant kernels and, for simplicity, let us put $N=1, S=$ $\sigma=0$, then we have

$$
\begin{aligned}
& u_{\boldsymbol{k}}(\boldsymbol{x}, 0)=[\tilde{G}(\boldsymbol{k})]^{1 / 2}[1-\tilde{\gamma}(\boldsymbol{k})]^{-1 / 2} e^{i \boldsymbol{k} \cdot \boldsymbol{x}}, \\
& \dot{u}_{\boldsymbol{k}}(\boldsymbol{x}, 0)=\left[-\frac{i}{2} \tilde{\boldsymbol{G}}(\boldsymbol{k})^{-1}\left[1-\tilde{\gamma}^{2}(\boldsymbol{k})\right]^{1 / 2}\right] u_{\boldsymbol{k}}(\boldsymbol{x}, 0) .
\end{aligned}
$$

For vanishing initial background field and momentum, standard equilibrium solutions are given by

$$
\begin{gathered}
G(\boldsymbol{k})=\frac{1}{2 \omega_{T}(\boldsymbol{k})} \tanh \frac{\omega_{T}(\boldsymbol{k})}{T}, \quad \omega_{T}(\boldsymbol{k})=\sqrt{\boldsymbol{k}^{2}+m_{T}^{2}}, \\
\gamma_{\boldsymbol{k}}=\left[\cosh \frac{\omega_{T}(\boldsymbol{k})}{T}\right]^{-1}
\end{gathered}
$$

with $m_{T}^{2}$ defined by the gap equation

$$
\begin{aligned}
m_{T}^{2}-m^{2}= & -I_{E}^{(1)}+\left(m_{T}^{2}-m^{2}\right) I^{(2)}(0)+\frac{1}{2} \lambda \int \frac{d^{3} k}{(2 \pi)^{3}} \\
& \times \frac{1}{2 \omega_{T}(\boldsymbol{k})} \operatorname{coth} \frac{\omega_{T}(\boldsymbol{k})}{2 T} .
\end{aligned}
$$

By expanding $\omega_{T}(\boldsymbol{k})$ around $\omega_{0}(\boldsymbol{k})=\omega(\boldsymbol{k})$ one can easily check the cancellation of divergent terms. We can then consider the out-of-equilibrium problem with the same initial conditions on the mode functions but arbitrary initial values for the background field and its velocity. The free mode functions read

$$
\begin{aligned}
u_{\boldsymbol{k}}^{(0)}(x)= & \frac{1}{2} \sqrt{\frac{1}{2 \omega_{T}} \operatorname{coth} \frac{\omega_{T}}{2 T}}\left[\left(1+\frac{\omega_{T}}{\omega}\right) e^{-i \omega t}\right. \\
& \left.+\left(1-\frac{\omega_{T}}{\omega}\right) e^{i \omega t}\right] e^{i x k} .
\end{aligned}
$$

Calculating the free correlation function we easily can check that the mean-field expression in the last of Eqs. (3.48) is free of divergences, except for the one at $t=$ 0 , which is given by Eq. (3.52) with $M=m_{T}$. As above, this can be cured by a Bogoliubov transformation, which in practice amounts to changing the equilibrium solution in Eq. (3.54) by allowing a suitable ultraviolet $k$ dependence in the temperature.

One last comment regarding RG invariance. We can see that the proof of Sec. III B still holds as long as the free correlation function depends on the renormalized squared mass alone.

\section{CONCLUSIONS}

In this paper we proposed an improvement of the HF approximation to the $O(N)$ scalar theory in the symmetric phase, motivated by its shortcomings as far as renormalizability and RG invariance are concerned. We first traced these shortcomings to the absence of the leading log contributions of a well-defined class of diagrams. We then set up a scheme in which these contributions are correctly included, while preserving the mean-field structure of the effective field equations and the one-loop approximation of the RG beta function. This implies a specific choice of momentum-dependent finite parts, which provide a sort of mean-field approximation of nonbubble diagrams such as the sunset diagram.

We were not concerned here with the accuracy of this approximation, for lack of space on one hand, and for general reasons on the other hand. In fact, as any meanfield approach, our proposal lacks direct particle scattering and therefore cannot describe properly many important phenomena of out-of-equilibrium QFT. Nonetheless, mean-field approximations provide good qualitative descriptions of many other phenomena and it is important, in our opinion, that they satisfy general field-theoretical requirements such as renormalizability and RG invariance.
[1] J. Berges, AIP Conf. Proc. 739, 3 (2004); J. Berges and J. Serreau, hep-ph/0410330; hep-ph/0302210.

[2] E. Manfredini, hep-ph/0101202.
[3] F. Cooper and E. Mottola, Phys. Rev. D 36, 3114 (1987); F. Cooper, S. Habib, Y. Kluger, E. Mottola, J. P. Paz, and P. R. Anderson, Phys. Rev. D 50, 2848 (1994). 
[4] D. Boyanovsky, H. J. de Vega, R. Holman, D. S. Lee, and A. Singh, Phys. Rev. D 51, 4419 (1995).

[5] J. Baacke and K. Heitmann, Phys. Rev. D 62, 105022 (2000); J. Baacke, K. Heitmann, and C. Patzold, Phys. Rev. D 57, 6406 (1998).

[6] C. Destri and E. Manfredini, Phys. Rev. D 62, 025007 (2000).

[7] G. Amelino-Camelia and S. Y. Pi, Phys. Rev. D 47, 2356 (1993).

[8] C. Destri and E. Manfredini, Phys. Rev. D 62, 025008 (2000).

[9] S. Michalski, hep-ph/0301134.

[10] T. Matsui, hep-ph/0111277; Y. Tsue, D. Vautherin, and T. Matsui, Prog. Theor. Phys. 102, 313 (1999).

[11] J. Berges and J. Cox, Phys. Lett. B 517, 369 (2001).

[12] S. Habib, Y. Kluger, E. Mottola, and J. P. Paz, Phys. Rev. Lett. 76, 4660 (1996); F. Cooper, S. Habib, Y. Kluger, and E. Mottola, Phys. Rev. D 55, 6471 (1997); B. Mihaila, T. Athan, F. Cooper, J. Dawson, and S. Habib, Phys. Rev. D 62, 125015 (2000).

[13] D. Boyanovsky and H. J. de Vega, Phys. Rev. D 47, 2343 (1993); D. Boyanovsky, H.J. de Vega, R. Holman, and J. F. J. Salgado, Phys. Rev. D 54, 7570 (1996); D. Boyanovsky, C. Destri, H. J. de Vega, R. Holman, and J. Salgado, Phys. Rev. D 57, 7388 (1998).

[14] C. Wetterich, Phys. Rev. E 56, 2687 (1997); L. M. A. Bettencourt and C. Wetterich, Phys. Lett. B 430, 140 (1998); G. F. Bonini and C. Wetterich, Phys. Rev. D 60, 105026 (1999).

[15] M. Salle and J. Smit, Phys. Rev. D 67, 116006 (2003); M. Salle, J. Smit, and J. C. Vink, Phys. Rev. D 64, 025016 (2001); G. Aarts and J. Smit, Phys. Rev. D 61, 025002 (2000).

[16] J. Baacke and S. Michalski, Phys. Rev. D 65, 065019 (2002).

[17] E. Calzetta and B. L. Hu, Phys. Rev. D 37, 2878 (1988).

[18] J. M. Cornwall, R. Jackiw, and E. Tomboulis, Phys. Rev. D 10, 2428 (1974).
[19] J. Baacke and S. Michalski, hep-ph/0312031; Phys. Rev. D 67, 085006 (2003); hep-ph/0409153; J. Baacke and A. Heinen, Phys. Rev. D 69, 083523 (2004).

[20] D. Dominici and U. M. B. Marconi, Phys. Lett. B 319, 171 (1993).

[21] J. Berges and J. Serreau, Phys. Rev. Lett. 91, 111601 (2003); J. Berges and M. M. Muller, hep-ph/0209026; G. Aarts, D. Ahrensmeier, R. Baier, J. Berges, and J. Serreau, Phys. Rev. D 66, 045008 (2002); J. Berges, Nucl. Phys. A699, 847 ( 2002).

[22] F. Cooper, J. F. Dawson, and B. Mihaila, Phys. Rev. D 71, 096003 (2005).

[23] J.T. Lenaghan and D. H. Rischke, J. Phys. G 26, 431 (2000).

[24] S. Y. Pi and M. Samiullah, Phys. Rev. D 36, 3128 (1987).

[25] F. Cooper, B. Mihaila, and J. F. Dawson, Phys. Rev. D 70, 105008 (2004).

[26] A. Jakovac and Z. Szep, Phys. Rev. D 71, 105001 (2005); hep-ph/0408360.

[27] J. P. Blaizot, E. Iancu, and U. Reinosa, Phys. Lett. B 568, 160 (2003); Nucl. Phys. A736, 149 (2004).

[28] H. van Hees and J. Knoll, hep-ph/0210262; hep-ph/ 0202263.

[29] J. Berges, S. Borsanyi, U. Reinosa, and J. Serreau, hep-ph/ 0503240.

[30] Y. B. Ivanov, F. Riek, and J. Knoll, Phys. Rev. D 71, 105016 (2005).

[31] O. J. P. Eboli, R. Jackiw, and S. Y. Pi, Phys. Rev. D 37, 3557 (1988).

[32] F. Cooper, S. Y. Pi, and P. N. Stancioff, Phys. Rev. D 34, 3831 (1986).

[33] F. Cooper, hep-th/9504073.

[34] K. c. Chou, Z. b. Su, B.1. Hao, and L. Yu, Phys. Rep. 118, 1 (1985).

[35] J. Baacke, K. Heitmann, and C. Patzold, Phys. Rev. D 55, 2320 (1997).

[36] J. Baacke, K. Heitmann, and C. Patzold, Phys. Rev. D 57, 6398 (1998). 